\title{
Evaluation of Functionalized Polymeric Surfactants for EOR Applications in the Illinois Basin
}

\author{
Larry Co ${ }^{a}$, Zijie Zhang ${ }^{a}$, Qisheng Ma ${ }^{a, b}$, Gary Watts ${ }^{a}$, Lin Zhao ${ }^{a}$, Patrick J. \\ Shuler ${ }^{\mathrm{a}}$, Yongchun Tang ${ }^{*}$, \\ ${ }^{a}$ ChemEOR Incorporation, 727 Arrow Grand Circle, Covina, CA 91722, U.S.A \\ ${ }^{b}$ Power Environmental Energy Research Institute, 738 Arrow Grand Circle, Covina, \\ CA 92711, U.S.A \\ * Corresponding Author, Email Address: tang@peeri.org (Y. Tang), (626) 858-5077
}

\begin{abstract}
Molecular modification of water-soluble hydrolyzed polyacrylamide (HPAM) with surfactantlike monomers, known as the functionalized polymeric surfactant (FPS), can be an effective enhanced oil recovery (EOR) method. The FPS-EOR operation is very similar to the conventional polymer flooding (P-flooding), in terms of the chemical use and field injection costs, but with a potential to further recover more than 5\% of OOIP compared to the HPAM-EOR alone. Laboratory tests and a third party core-flood result show FPS can recover more oil than HPAM even at the lower injection pressure. Surfactant-like monomers linked to the FPS backbone improve the microscopic displacement efficiency of water-soluble polymer by pulling them towards the oil-water interface and creating an oil-water emulsion. Unlike the conventional surfactant + polymer $(S+P)$ multi-component systems, single-component FPS-EOR injection has both the sweep efficiency (polymer feature) and the microscopic displacement efficiency (surfactant feature), but can mitigate incompatibility issues such as chromatography separation and surfactant-polymer interactions. Unlike a typical surfactant flooding for which the reduction of oil-water interfacial tension (IFT) to an ultralow level $\left(<10^{-3} \mathrm{dyne} / \mathrm{cm}\right)$ is required, a typical FPS solution only reduces IFT to a moderate level $\left(\sim 10^{-1} \mathrm{dyne} / \mathrm{cm}\right)$ at the polymer concentrations of $1000 \sim 3000$ ppm.
\end{abstract}

KEYWORDS: Functionalized Polymeric Surfactant, Chemical Enhanced Oil Recovery, Polymer Flooding, Water External Microemulsion, and Interfacial Tension.

HIGHLIGHTS:

- A FPS flooding can recover more oils than the conventional polymer flooding;

- Implementation of single-component FPS flooding is as easy as polymer flooding;

- Molecular modification of FPS can improve its EOR ability;

- Formation of the water external emulsion indicates good FPS-EOR efficiency. 


\section{INTRODUCTION}

Chemical Enhanced Oil Recovery (EOR) processes involve the injection of a fluid with some types of chemical into a reservoir to assist oil flowing to the production well. The viability of a successful EOR process is dependent on two important factors: volumetric sweep efficiency and microscopic displacement efficiency. Polymer flooding (P-Flooding) is one of the most widely applied mobility control processes, with the main purpose to develop a more uniform sweep of the reservoir. Inclusion of the high molecular-weight polymers, e.g., Polyacrylamide (PAM) or partial Hydrolyzed PAM (HPAM), thickens the injection fluid, so as to prevent water from fingering through the oil and moving only through the most conductive flow paths to the production well. The traditional P-Flooding operations suffer from the low oil recovery efficiency $(<10 \%$ of OOIP, Original Oil-in-place), because it does not have significant microscopic displacement efficiency. The water-soluble polymers remain inside the aqueous solution, which limits their interactions with the oil phase as a free fluid in the pore spaces, or as it may be adhered to the reservoir rock surfaces. For example, Gogarty [1] studied the rheological properties of non-Newtonian fluids in porous media and the effects of the effective viscosity by addition of the high molecular weight polymer for the oil recovery. Manning et al. [2] analyzed statistical data of the field-wide EOR projects, and concluded that the median oil recovery of P-Flood was $2.91 \%$ OOIP. Schurz et al. [3] summarized results from 99 P-Flooding projects initiated during 1980-1989 and the projected median incremental oil recovery ranges between $3.7 \%$ and $4.8 \%$ OOIP.

Modification of the association and self-assembling ability of PAM/HPAM to improve viscosity for EOR applications has been intensely investigated [4,5]. McCormick et al. conducted a fundamental research program in lab with the ultimate goal of developing "smart" multifunctional polymers that can respond in-situ to stimuli and result in significantly improved sweep efficiency in EOR processes [6]. The main purpose of incorporating a small fraction of hydrophobic monomer into an HPAM polymer is intended to generate efficient and stable viscosity to improve sweep efficiency as mobility controllers. However, such an enhancement in viscosity through polymer association is significantly dependent on the polymer concentrations, operational shear rate, and the temperature, $\mathrm{pH}$ and salinity of reservoir; the complexity of having several variables impacting performance has become the key issue inhibiting widespread applications of associative polymers for EOR [7].

The multi-stage injection scheme of using a combination of surfactant and polymer (Surfactant + Polymer, SP-Flooding) has been the conventional EOR practice to achieve a substantial improvement in both sweep and microscopic displacement efficiency. In this process, a primary surfactant slug is injected [8,9], to achieve very low interfacial tension (IFT) for improved displacement efficiency, followed by a polymer solution to maintain favorable mobility control and improved sweep efficiency. 
Influenced by the conventional wisdom of employing ultralow IFT displacing fluid in the chemical process, attempts of using polymeric surfactant (PS) for the EOR applications have been mainly aimed at lowering IFT with the PS-containing fluids. Chen et al. specifically emphasized that the water-oil IFT should be less than $0.1 \mathrm{dyne} / \mathrm{cm}$ (preferred $0.005 \mathrm{dyne} / \mathrm{cm}$ ) in order to achieve an optimum microscopic displacement efficiency of the aqueous fluid containing the polymeric surfactants [10]. In order to achieve low or ultra-low IFT, certain surfactants, co-surfactant and sometimes oil and alkaline were added with the PS-containing aqueous solutions. For examples, Cao et al. [11] identified a novel family of PS which might have potential for enhance oil recovery. Under the influence of added alkali, the IFT of PS in aqueous solution, decreases to the level of less than $10^{-2}$ dyne/cm. Elraies and Tan described a chemical process to attach the sulfonate group of Sodium Methyl Ester Sulfonate (SMES) to the PAM-backbone to produce polymeric methyl ester sulfonate (PMES), and examined their EOR applications as one component system for IFT reduction and viscosity control [12]. Certain amounts of alkali were present from the raw materials or as additives to examine the IFT effects of alkali. However, as being pointed out by the authors, the rapid decrease in the IFT value can be attributed to the production of in-situ surfactants due to saponification reactions between the alkali and the acidic groups in the crude oil. There are drawbacks, however, by introducing noticeable alkali amounts into a reservoir. Similar to the well-studied Alkali + Surfactant + Polymer (ASP-Flooding) processes, alkaline often generates extremely tight emulsions which are difficult to break; the waste water treatment is much more difficult; and it also causes additional scale deposition issues. It is therefore, economically risky and operationally intensive in EOR operations, even though ASP often shows great promise from laboratory core-flood experiments and some field tests.

Our strategy is to develop a special class of PS designated as Functionalized Polymeric Surfactant (FPS), a single-component having the right balance of better volumetric sweep and microscopic displacement efficiency. Our FPSs are made through same processes of PAM/HPAM polymers with the addition of certain amounts of specifically selected surfactantlike monomers. Therefore, the FPS-EOR operation will be just like normal P-Flooding to increase the fluid viscosity therefore increasing the sweep efficiency. In addition, the surfactant feature of a FPS can also overcome the capillary force between oil-water interfaces. For conventional surfactant solution, the IFT reduction is often related to the emulsification ability (the low IFT, the higher the ability to emulsify oil). But for the FPS solution, it is not always the case. For example, our FPS systems exhibit sufficient emulsification potential, even though they do not have ultra-low IFT. Such emulsifications, unlike those created by the alkaline additives, are relatively easily to be broken with time.

Typically the water external emulsions at the oil-water interface have slightly higher viscosity than the bulk viscosity of FPS-containing water. The higher viscosity of the water external emulsion caused by FPS at the oil-water interface can help exert an additional force on oil under reservoir condition (increasing capillary number). Of course, if a FPS forms an oil external emulsion with too high of viscosity, it would not be a good candidate for EOR. Thus a 
good FPS candidate can provide reasonable water viscosity and strong emulsion ability. This can lead to a decrease of the oil saturation even at the medium range oil-water IFT value, such as in 0.1 to $15 \mathrm{dyne} / \mathrm{cm}$ range [13]. The single-component FPS-EOR process can be an ideal replacement of the low-efficiency P-Flood operation with increasing oil recovery; and it can also effectively replace traditional multiple components of SP-Flood or complicated ASP-Flood oil recovery methods, both expensive and difficult to apply, especially for small oil producers.

\section{ILLINOIS BASIN}

Considering FPS-EOR as one of the most practical improved oil production approaches for small oil producers who manage production from mature oil fields, we emphasize demonstrating the technology opportunity for this technology in the Illinois Basin. The Illinois Basin is an asymmetrical shape structural depression that trends northwest-southeast and it filled with more than 14,000 feet of Paleozoic sediment at its deepest point. Oil productions from the Illinois basin have a long history, with production peaks in 1908 and 1940 by primary recovery, and the third peak in 1960 from implementation of secondary water-flooding technology $[14,15]$. However, the oil production from the Illinois Basin has gradually declined in the past 60 years, and it has a recent low of 51 thousand bbl/d in 2004.

Nevertheless, the Illinois Basin still holds a significant amount of petroleum resource in the subsurface, with over 7.5 billion barrels of oil that are still unproduced, due to the lack of advanced recovery technology, high associated cost, and high economic and technical risks (Table 1) [16]. In addition, because it is dominated by very mature water-flooded reservoirs with small pattern spacing containing light crude oil, the Illinois Basin has especially high potential for advanced EOR applications [17]. At the current date, there are virtually no major energy companies actively pursuing oil production in the Illinois Basin. Most of the oil producers are small and/or medium in size with limited capital and resources to commit to the large-scale EOR project. The simplest P-Flood and FPS-EOR operations could be easily implemented and therefore be more attractive to smaller oil producers.

Illinois Basin is quite shallow (1000 4000 feet) and the reservoir temperature are typically low $\left(60 \sim 100 \mathrm{~F} / 15^{\sim} 37^{\circ} \mathrm{C}\right.$ ); it has more sandstone ( 75\%) than carbonate ( $25 \%$ ) oil reservoirs. These make the conventional P-Flood and/or FPS-EOR superior to other alternatives such as $\mathrm{CO}_{2}$-EOR. The major oil/gas pay zones are located in three geologic systems:

The Pennsylvanian Sandstones generates approximately $13 \%$ of the basin's oil production. About $40 \%$ of this production has come from sandstone lying at or near the base of the system; while $60 \%$ occurs in fluvial-deltaic sandstone higher in the Pennsylvanian sequence. The pay zones are often referred as Penn Sands or Pennsylvanian Sands, or locally as the Robinson, Biehl, Buchanan Sands, and others in Indiana and Kentucky.

The Upper Mississippian/Chesterian Series consists of a cyclical arrangement of Chesterian carbonate, shale and sandstone strata resulted from repeated advances and retreats of the ocean across a southward dipping, slowly subsiding coastal plain. These are mostly fine-grained 
and well sorted sandstone deposited as point bars, offshore bars and distributary channels, which contain $\sim 60 \%$ of the oil production. The pay zones in ascending order are: Renault, Benoist, Cypress, Jackson, Hardingsburg, Tar Springs, Waltersburg, Palestine, and Degonia-Clore Sandstones.

The Mississippian/Valmeyeran Series consists of limestone (Salem, St Louis, and St. Genevieve) and Aux Vases Sandstone (fine/very fine quartz sandstones) that are deposits on a shallowmarine shelf dominated by tidal processes. Unlike the other two formations, their pay zones are less suitable to FPS-EOR applications as the deposition can be in cross disconnected layers and compartmentalized zones.

A report including approximately 500 samples from the area south of the base line to the third principal meridian generally pictured the field crude oil properties in Illinois Basin (Figure 1, Figure 2). The base line nearly across the middle of the Illinois Basin goes approximately from Belleville to Mt. Carmel. The data from the report show that most field oils in Illinois Basin are light, with a majority API gravity of 37 and viscosity of $3 \mathrm{cp}$ at $100^{\circ} \mathrm{F}$ [18-19].

A total of 13 crude oil and brine samples from 4 different locations (Gibson County and Posey County in Indiana, Henderson County and Vanderburg County in Kentucky) of the Illinois Basin (Figure 3) have been collected for detailed laboratory evaluation of the developing FPSEOR technology. Among them, 4 are from Pennsylvanian Sandstone formations, 8 from Mississippian Chesterian, and 1 from Mississippian Valmeyeran formations. Oil/Brine samples were collected either from flowing oil and water into storage tanks, or directly from pumping oil wells (Table 2).

Tank collection was accomplished by attaching a 250 milliliter plastic sampling bottle on an extension device and locating it under the incoming stream of oil or water. Oil samples were allowed and encouraged to degas so as to provide an inert oil sample. Samples collected directly from the pumping oil wells presented a more challenging methods to separate, due to very high water cut (approximately 99\% water and 1\% oil). Large quantities of raw samples (at least 6 gallons) were required in order to extract sufficient amount of oil samples.

Chemical composition of the formation water from different wells vary significantly. For the screening and comparison purposes, we used the synthetic model formation water in most of the studies. The compositions of our synthetic formation water are listed in Table 3. Such a synthetic brine composition is based on the replicate sampling and analysis of the formation water from a single well in Tilden field of the Illinois Basin (Table 4) [20].

\section{EXPERIMENTAL METHODS}

Emulsification - Phase behavior of different FPS in association with crude oil at different water salinities is studied qualitatively in the course of this study. Typically, 4 grams of the $2000 \mathrm{ppm}$ FPS solution and 4 grams of the crude oil are added in a test tube. The test tube is capped and 
shaken at 80 strokes/min for 10 minutes, then allowed to stand for 30 minutes. The volume of the emulsification results is then recorded.

Viscosity - Two Brookfield-type digital viscometers (Brookfield DV-II + Pro, and DV-I prime digital viscometers) have been used to measure the viscosity. Both of them are featured by variable speed capability (0.01-200 rpm for DV-II + Pro, and 3-100 rpm for DV-I prime digital), with a continuous display of temperature, viscosity and shear stress, and have repeatability of $0.2 \%$ of full scale range. Viscosity measurements are performed at $6 \mathrm{rpm}$ and $30 \mathrm{rpm}$, approximately a shear rate of 8 and 40 sec-1, respectively).

Interfacial Tension (IFT) - Spinning drop method is used for the IFT measurements. This technique is ideal for measuring low IFTs. The diameter of a drop within a heavy phase is measured while both are rotated, and the IFT value can be calculated based on the diameter. Two digital spinning drop tensiometers (TX-500C full range) were used in measuring IFT at a specified temperature and a specified rotation ( $\mathrm{rpm}$ ) value. The measurements for different FPS solutions and crude oils are done at different time intervals (5, 10, 20, 40, 60 and 100 minutes) at the $5000 \mathrm{rpm}$ rotation speed. The typical FPS concentration is $0.2 \mathrm{wt} \%$ and the temperature is at $50{ }^{\circ} \mathrm{C}$.

Thermal Stability Test - The FPS solutions are prepared and placed into sealed vials that are pre-flushed with nitrogen. A series of vials containing the FPS solutions are placed inside an oven held at a given temperature (e.g. $80^{\circ} \mathrm{C}$ ). Vials are taken out one at a time at selected intervals, and the viscosity of the FPS solution is measured. A decreased viscosity indicates the thermal instability of the FPS solution.

Coreflood - The core-flooding systems consists of similar components as in the sand-pack system, except that the sand pack column is replaced by the sealed core holder. The Berea sandstone core with $300 \mathrm{mD}$ permeability and $21.4 \%$ porosity is first heated to $120{ }^{\circ} \mathrm{C}$ for 3 hours, then inserted into the rubber tube inside a closed column. The column surrounding pressure is raised to 100 psi through filling with deionized water. The inner core column is placed under high vacuum for 6 hours and saturated with synthetic Illinois brine for overnight. Absolute permeability is determined by injecting the brine solution at a constant flow rate. The water-flooding is conducted until the water-cut achieved is above $99 \%$, followed by the FPSflooding or the regular HPAM-flooding (1.5 Pore Volume, PV) and the chasing water flooding (1 PV). Temperature is maintained at $38{ }^{\circ} \mathrm{C}$ which is close to Illinois Basin conditions during the core flooding. All recovered fluids are collected and analyzed to determine the oil recovery performance. That is, after setting for a period of time, the recovered fluids self-separate into oil and brine two layers. The oil recovery is calculated by the oil volume read in the upper part of the vial divided by the initial total oil saturation.

To further verify our results, core flood test was also performed for HPAM and FPS by a third-party standard laboratory. Fired Berea sandstone with $21.4 \%$ BV and 73.41 volume was used in tests. 


\section{RESULTS AND DISCUSSION}

\subsection{Viscosity of FPS}

Viscosity of the FPS solution has been considered as one of the most important parameters for EOR applications. Numerous viscosity tests have been performed to characterize viscosity of FPS solutions under different conditions. A typical viscosity curve of the FPS solution versus FPS concentration is depicted in Figure 4. As expected, at the elevated temperature, a higher FPS concentration is required to achieve the same viscosity as in the lower temperature. For consistent comparisons, the FPS concentration has been maintained at the $2000 \mathrm{ppm}$ level unless otherwise stated. The effects of salinity $(\mathrm{KCl} \%)$, shear rate, and temperature on the selected FPS solutions are depicted in Figure 5 . The results show that the viscosity of FPS can persist at an acceptable level at a mild salinity (Figure 5(a)), mild shear rate (Figure 5(b)), and targeted temperature (Figure 5(c)). For instance, higher salinity causes the polymers to coil, showing a lower viscosity, but is not noticeable in following even higher salinities. Similarly, the viscosity of FPS is not sensitive to the mild shear rate. Note that the increasing percentage of surfactant monomers can sharply decrease the viscosity of FPS solution (Figure 6), which indicates an optimized balance needs to be met between the sweep efficiency represented by viscosity and the micro displacement effect controlled by the surfactant monomers for recovering more oil.

\subsection{IFT of FPS}

Oil-Water IFT is a critical measure of tertiary oil recovery potential for conventional surfactant EOR methods. A surfactant is capable of lowering oil-water IFT, due to its partial partitions in both the oil and water phases. In general, the IFT value changes with respect to treated crude oils and the water quality. A FPS can also lower oil-water IFT when the surfactantlike monomers are incorporated into its molecular backbone. IFT values of the FPS solutions will also change with different crude oils (Figure 7) and in various water salinity (Figure 8). It is worth noting that the typical HPAM solution has an oil-water IFT in the range of $20 \sim 30$ dyne/cm. Thus most of the FPS systems studied can lower the oil-water IFT to 1 to 2 orders of magnitude versus HPAM, even though it cannot achieve the ultra-lower ( $\left.10^{-3} \mathrm{dyne} / \mathrm{cm}\right)$ IFT such as in the surfactant solution. Among the oil samples tested with FPS, the IFT of \#11 outstands with a result below $0.15 \mathrm{dyne} / \mathrm{cm}$. From another test for the crude oil samples, the viscosity of the \#11 crude oils at $50{ }^{\circ} \mathrm{C}$ and $30 \mathrm{rpm}$ with Brookfield viscometer is remarkably high (Figure 8), indicating a different crude oil property.

\subsection{Solubility of FPS}

Dissolution of FPS in the aqueous solution plays an important role in the EOR applications. Hydrophobic modification of PAM-based FPS can effectively improve their oil recovery performance, however, their water/brine solubility might be reduced. For example, as shown in 
Figure 10, the insolubility of the FPS solution often results in the formation of insoluble gels. FPS versions that form gels in the target reservoir or injection brine are not suitable for that particular location. Only FPS compositions that are soluble in the brines of interest and at the reservoir temperature are viable candidates for that particular location.

\subsection{Emulsification of FPS}

Oil-in-water emulsions are formed typically when the Illinois crude oils and synthetic formation water are mixed with the 2000 ppm FPS solutions (Figure 11). The straight line shows the baseline of the oil-water interface between emulsions. This special FPS solution (FPS) has potential to form volume of emulsion with all tested Illinois crude oil samples, wherein it reaches the optimized emulsification performance towards the \#6 oil as shown.

To further illustrate different emulsions formed by the FPS solution, we also used a pure hydrocarbon, dodecane $\left(C_{12}\right)$, to model the oil phase; whereas the aqueous phase contains different salinities modified with various $\mathrm{KCl} \%$ concentrations (Figure 12 ). In contrast to the Illinois crude oil systems, we observed formation of a mixed layer in the upper section of the tube. On the most left side in Figure 12, the water-oil interface without the addition of FPS is shown. Unlike the emulsification tests of the crude oils, there are not significant changes of the volume of water, regardless of the water salinity, suggesting that the worse miscibility of the modeled oil compound with water than that of the crude oils.

When the oil-in-water emulsion is formed, wherein the oil is the dispersed phase and water is the dispersed medium, we also call it the water-external emulsion. Substantial amount of oil is extracted into the water phase, facilitating oil recovery. On the other hand, when the waterin-water emulsion is formed, wherein the water is the dispersed phase and oil is the dispersion medium, we can call it the oil-external emulsion. These emulsion forms can be viewed and distinguished microscopically, by directly observing through a lighted microscopy a drop of the emulsified mixture (Figure 13). In the water-external emulsion (Figure 13(a)), oil droplets (darker circles) are formed, whereas in the oil-external emulsion (Figure 13(b)), water droplets (whiter circles) are formed. For the emulsion of crude oils with the FPS solution, a waterexternal emulsion (Figure 13(c)) is observed.

\subsection{Core-flood of FPS}

Core-flood tests are essential for laboratory evaluation of the EOR ability of candidate chemical solutions. The FPS-J solution was selected for the core-flooding test of the \#6 Illinois crude oil. For comparison, a regular HPAM solution (Polyflood ${ }^{\mathrm{TM}} \mathrm{Max}^{-165}$ ) $^{1}$ is also used (Figure 15). After the initial water flooding and the water-cut achieved was above $99 \%$, the injection of the 1.5 PV of 1500 ppm of either HPAM or FPS-J solution both recovered additional $13 \sim 14 \%$ OOIP. However, the follow-up water drive flood to ensure a full recovery of residual HPAM/FPS from cores did show a significant difference. An additional $4.5 \%$ OOIP was recovered from the

\footnotetext{
${ }^{1}$ Polyflood $^{\mathrm{TM}}$ is a trademark of ChemEOR Inc.
} 
water-drive following the FPS-J flood, while there is almost no additional recovery from the water-drive flood following the HPAM flood. Consequently, the overall oil recovery $(18.04 \%$ OOIP) from the FPS-J flooding is considerably higher than from the regular HPAM (13.43\% OOIP) flooding (Figure 14).

Similar results were observed from a core-flood test performed by a third-party standard laboratory (Figure 15). A regular HPAM solution (Polyflood ${ }^{\mathrm{TM}}$ Max-165) ${ }^{1}$ and the FPS-J solution were selected for the core flooding test comparison. Crude oil used is from East Texas and the test temperature is maintained at $80^{\circ} \mathrm{C}$. After the initial water flooding recovering $45.47 \%$ OOIP, HPAM flooding drove out $4.13 \%$ additional oil from the core, followed by water chase which also recovered $0.14 \%$ OOIP. Consequently, a total of $4.27 \%$ OOIP has been recovered for HPAM flooding and water chase (Figure 16(a)). On another run, FPS-J followed by the water chase recovered a total of $6.91 \%$ additional oil after water flooding pushing out $53.01 \%$ initial crude oil (Figure 16(b)), with the FPS-J flooding and water chase recovered $6.48 \%$ and $0.43 \%$ additional oil, respectively.

In this set of run, the pressure changes in between the pumping pump and the core were continuously measured and presented in the figures as well. In the initial water flooding process, the pressures are maintained at $\sim 5$ psi for both cores. For the sequential HPAM polymer flooding, the pressure was raised up to 19.19 psi (3.55 times of the water flooding pressure), then dropped to 15.01 psi after breakthrough (21.78\% decrease from the peak value). Whereas for the FPS flooding, the highest pressure was set up 10 psi (1.85 times), then plunged to $5.1 \mathrm{psi}$ in water chase step (49\% decrease). The higher pressure drop in the FPS-flooding case is probably due to the lower viscosity of FPS (60 cps at $80^{\circ} \mathrm{C}$ ) compared to $21.8 \mathrm{cps}$ of HPAM under same conditions. Consequently, one would expect the higher sweeping efficiency of the regular HPAM flooding, compared to that of the FPS flooding. However, the total oil recovery from the FPS flooding is even higher than that from the HPAM flooding, suggesting that another oil recovery factor (e.g., oil displacement efficiency) will be responsible for the high oil recovery in the FPS flooding. The final pressure of the FPS flooding at $5.1 \mathrm{psi}$ is nearly equal to the original 5 psi, compared to the 15.01 psi in the HPAM case, suggesting that the residual FPS in cores is negligible (either recovered or decomposed). Therefore, one would expect a quick permeability regaining and less formation damage in the FPS flooding case.

\section{CONCLUSION}

A FPS polymer may be formed through structural modification of the regular water-soluble HPAM polymer by incorporating a small amount of surfactant-like monomers into the chain structure. Application of the FPS solution as an advanced polymer flooding chemical for EOR are discussed in this paper. Even though a FPS solution does not have the ultra-low oil-water IFT values, the oil recovery potential of FPS flooding could be improved substantially as compared to ordinary HPAM flooding. Because only a small amount (typical 1 - $5 \mathrm{wt} \%$ ) of the functional monomer is used in the FPS synthesis procedure, the associated manufacturing cost of FPS will be comparable to that of common commercial HPAM products. Also because the FPS is a 
single-component chemical, the field operation and injection of the FPS flooding will be very similar to that of the ordinary polymer flooding. An additional 5\% OOIP could be recovered via FPS flooding versus that from HPAM flooding. Such a recovery efficiency might not be comparable with the well-known Surfactant + Polymer flooding (S+P) EOR operation, however, the lower-cost and simplified implementation of FPS-EOR will be well-suited for the small- or medium-size energy producers, such as those in the Illinois fields.

The special features of the developed FPS chemical should include (1) the ability to emulsify oil to form an emulsion; (2) can also reduce oil-water IFT to a moderate value $\left(10^{-2} \sim 10^{-1}\right.$ dyne/cm); (3) can increase the solution viscosity at reasonable polymer concentration (1000 $3000 \mathrm{ppm}$ ); and (4) recover additional oil with surfactant like monomer at low injection pressure. Unlike the regular HPAM polymer, which has only the capability to be a mobility control agent, the surfactant-feature of the FPS polymer can also directly interact with crude oil to provide additional oil recovery.

\section{ACKOWLEDGMENT}

Part of this work was financially supported through from US Department of Energy through the Research Partnership to Secure Energy for America (RPSEA) Small Producer program, under the contract agreement number 10123-03. We would like to thank Ms. Lin Zhao and Mr. Chao Chen from ChemEOR for their assistances on synthesis and characterization of different FPS chemicals. Fruitful and stimulating discussions with Dr. Carl W. Aften and Dr. Dons Wicks also have contributed significantly to the present works.

\section{REFERENCES}

[1]. Gogarty, W. B. (1967) Rheological Properties of Pseudoplastic Fluids in Porous Media, SPE1566-A. SPE Journal, $149-160$.

[2]. Manning R. K., Pope G. A. and Lake L. W. (1983) A Technical Survey of Polymer Flooding Projects. US Department of Energy DOE/BETC/10327-19, Bartlesville, Oklahoma.

[3]. Schurz G. F., Martin, F. D., Seright, R. and Weiss, W. W. (1989) Polymer-Augmented Waterflooding and Control of Reservoir Heterogeneity, NMT 890029, New Mexico Tech Centennial Symposium.

[4]. Taylor, K.C. and Nasr-El-Din, H.A. 1998. Water-Soluble Hydrophobically Associating Polymers for Oil Recovery: A Literature Review. Petroleum Sci \& Engineering 19. 265-280.

[5]. Wei B., Romero-Zeron L, and Rodrigue D. (2014) Novel Self-assembling polymeric system based on a hydrophobic modified copolymer: formulation, rheological characterization, and performance in enhanced heavy oil recovery. Polym. Adv. Technol. 25(7), 732-741.

[6]. McCormick C. L. et al. (2004) (2005) Smart Multifunctional Polymers DOE Final Report DEFC26-03NT15407. 
[7]. Seright R. S., Fan T., Wavrik K., Wan, H. Gaillard N., and Favero C. (2011) Rheology of a New Sulfonic Associative Polymer in Porous Media. SPE 141355, SPE International Symposium on Oilfield Chemistry, Woodlands, TX.

[8]. Iglauer, S. Wu, Y., Shuler, P., and Tang, Y. (2010) New Surfactant Classes for Enhanced Oil Recovery and Their Tertiary Oil Recovery Potential. J. Petro. Sci. \& Eng. 71, 23-29.

[9]. Wu, Y. Shuler, P., Blanco, M., and Tang, Y. (2008) An Experimental Study of Wetting Behavior and Surfactant EOR in Carbonates with Model Compounds, SPE Journal 13(1), 26-34.

[10]. Chen, C. S. H. and Sheppard, E. W. (1981) Oil Recovery by Waterflooding Employing Anionic Polymeric Surfactants. US-Patent 4,284,517; 4,317,893.

[11]. Cao, Y. and Li, H. (2002) Interfacial Activity of a Novel Family of Polymeric Surfactants. European Polymer Journal 38(7), 1457-1463.

[12]. Khaled Abdalla Elraies and Isa M. Tan (2012). The Application of a New Polymeric Surfactant for Chemical EOR, Introduction to Enhanced Oil Recovery (EOR) Processes and Bioremediation of Oil-Contaminated Sites, Dr. Laura Romero-Zerón (Ed.), ISBN: 978-953-510629-6, InTech.

[13]. Wang, D., Cheng, J., Xia, H., Li, Q., \& Shi, J. (2001). Viscous-Elastic Fluids Can Mobilize Oil Remaining after Water-Flooding by Force Parallel to the Oil-Water Interface. Society of Petroleum Engineers. doi:10.2118/72123-MS.

[14]. Swann D. H. (1968) A Summary Geologic History of the Illinois Basin, in Geology and Petroleum Production of the Illinois Basin, Illinois Geologic Society, p.3-21.

[15]. Ridgley J. (2009) A Rebirth of the Illinois Basin, US Geological Survey, retrieved 29 October 2009.

[16]. Lewis, J. and Bergeron, L. (2010) 'The economic impacts of the energy industry in Illinois', Regional Development Institute, Northern Illinois University, Dekalb, Illinois ISCC Energy Industry Report No. 7-12-2010, pp.3-40.

[17]. Scott M. Frailey, ' $\mathrm{CO}_{2}$ Enhanced Oil Recovery in the Illinois Basin', Illinois State Geological Survey, April 2012.

[18] Armon W. J., Coburn A. A. Mast R. F., and Sherman C. W. (1964) Physical Properties of Illinois Crude Oil, Part I, Illinois Petroleum 78, Illinois State Geological Survey, Urbana, Illinois.

[19] Armon W. J., Lawry T. F. and Mast R. F. (1966) Physical Properties of Illinois Crude Oil, Part II, Illinois Petroleum 81, Illinois State Geological Survey, Urbana, Illinois.

[20]. Stueber A. M. and Walter L. M. (1991) Origin and Chemical Evolution of Formation Water from Silurian-Devonian Strata in the Illinois Basin, USA. Geochimica et Cosmochimica Acta, 55, 309-325. 


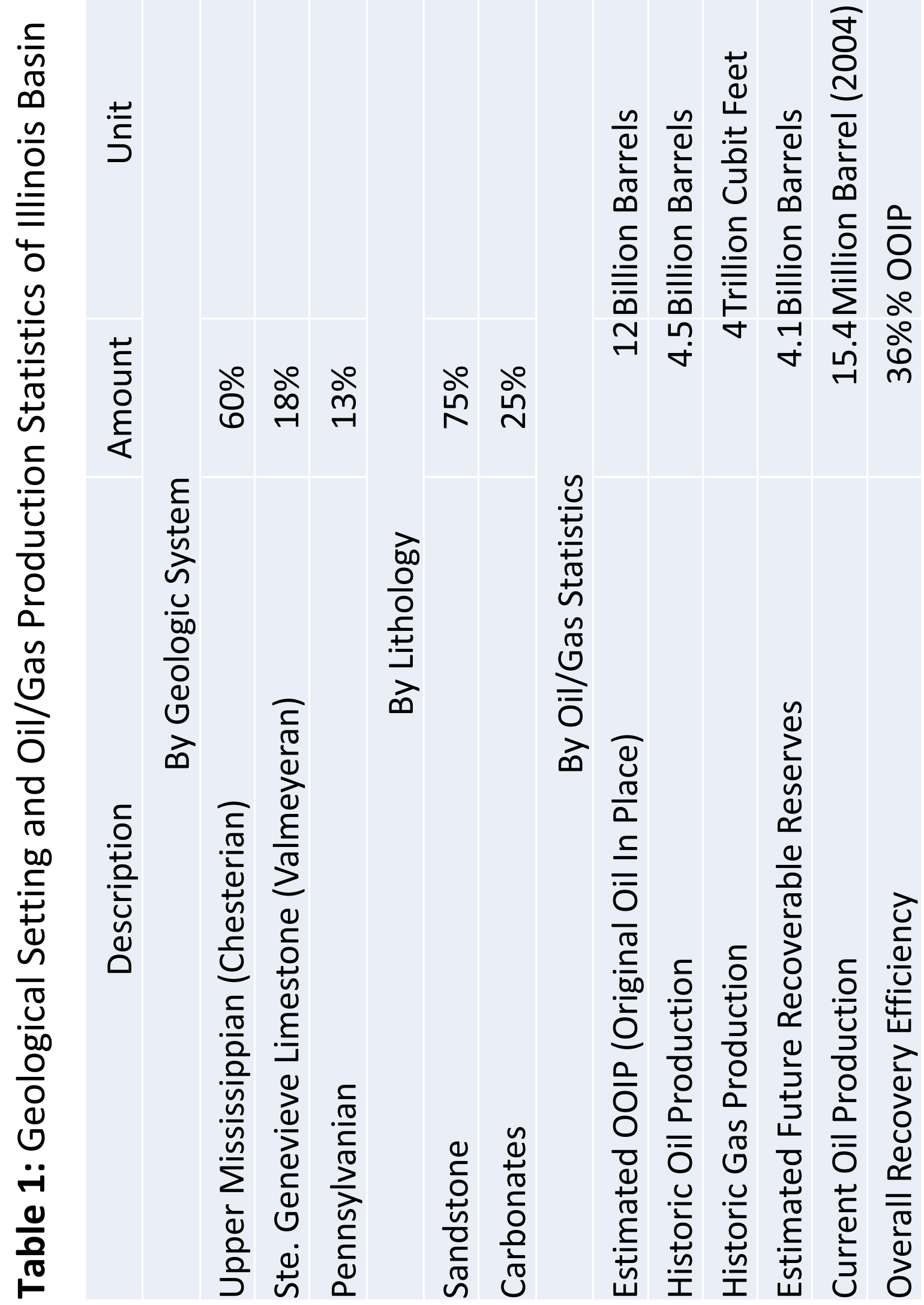




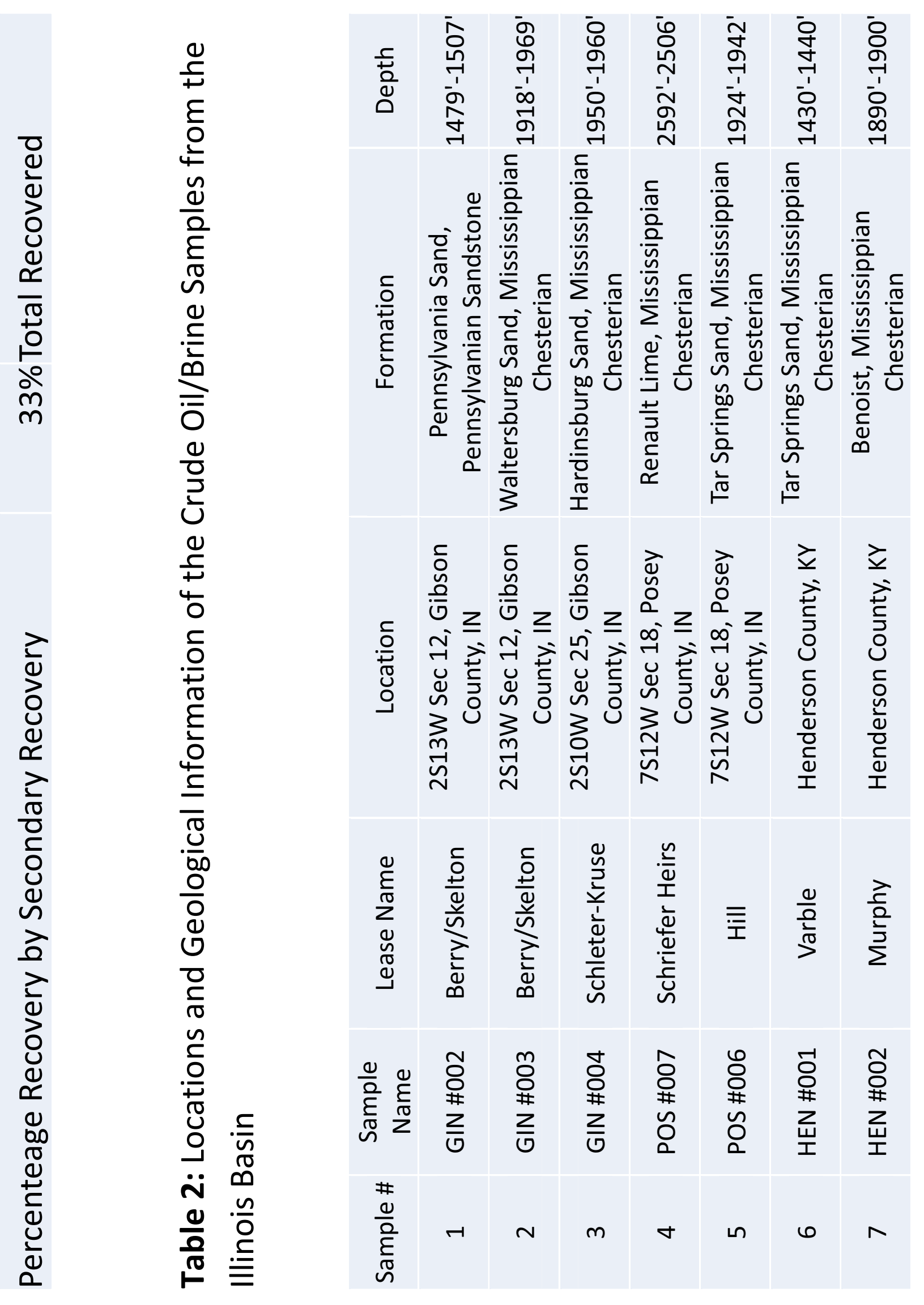




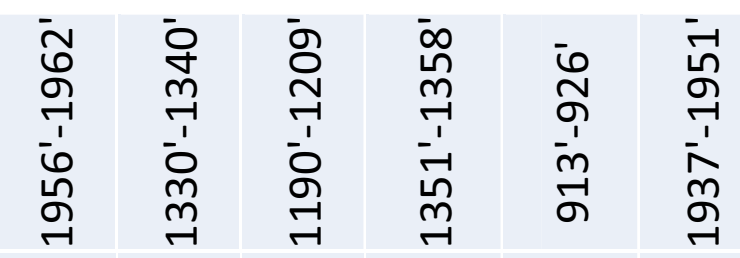

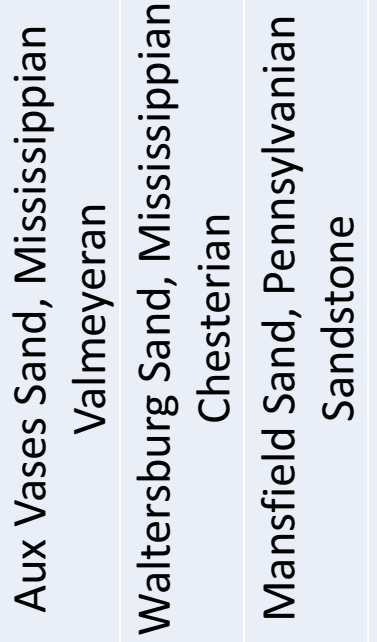

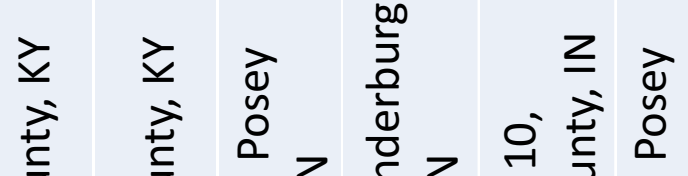

竎

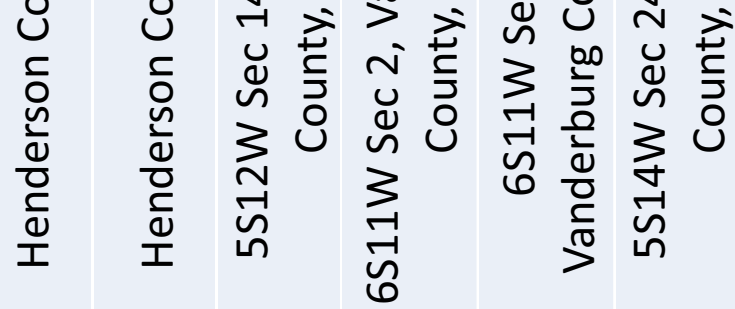

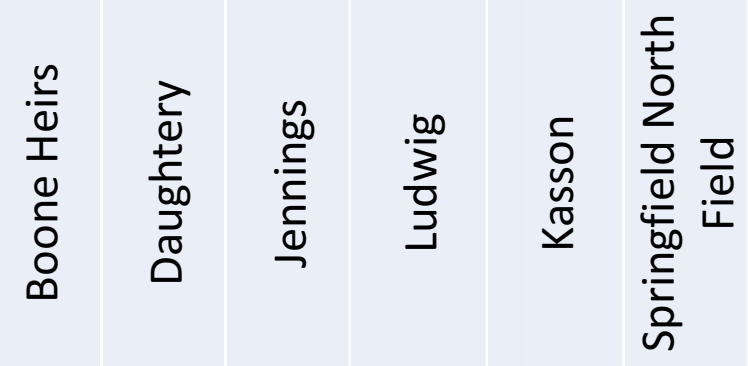

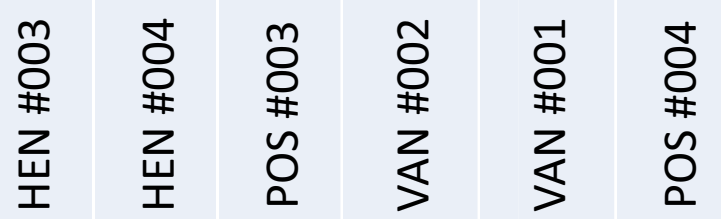

$\infty a$ 의 $\cong m$
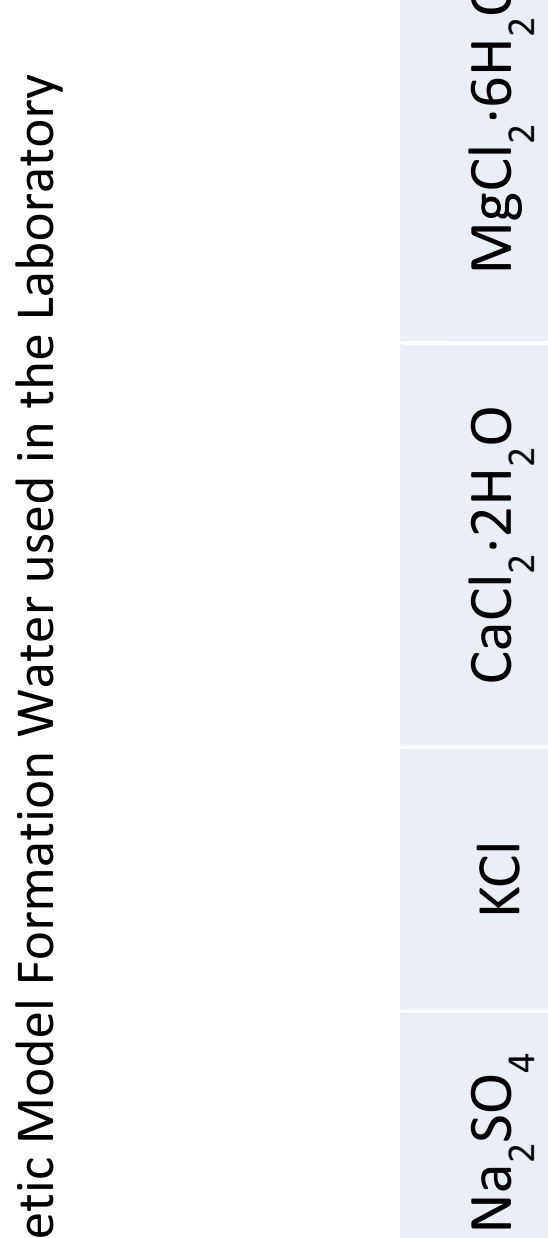

$\bar{\mho}$

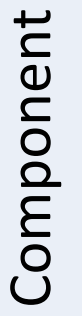


$\begin{array}{ll}m & 8 \\ \text { m } & \text { gి } \\ & \text { न्न }\end{array}$

$\overbrace{\text { ก }}^{2}$

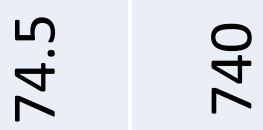

$\underset{\forall}{N} \stackrel{9}{0}$

20

$\stackrel{\square}{0}$

$\stackrel{0}{3}$

$\frac{1}{\sqrt{2}}$

$\frac{0}{0}$

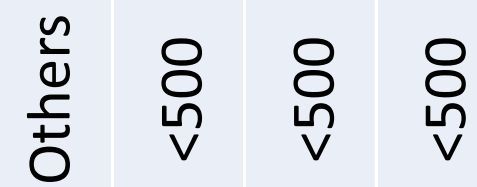

ن்

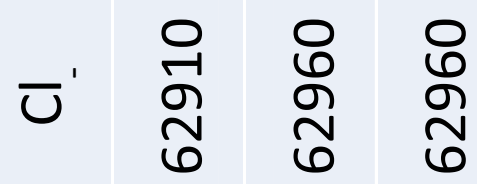

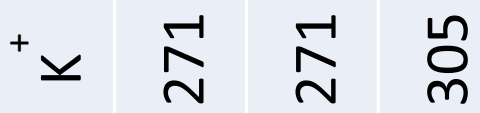

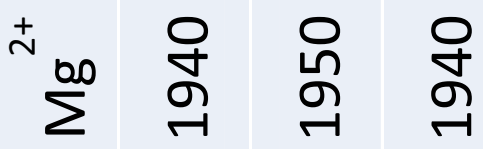

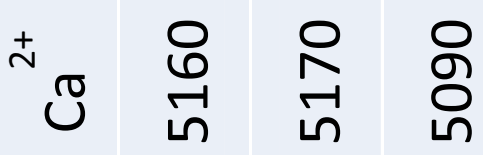

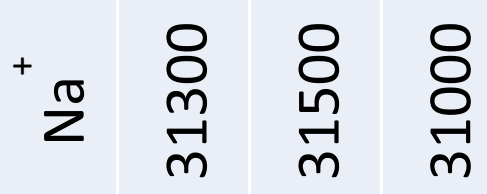

$\begin{array}{lll}\sim & m\end{array}$

$\stackrel{\oplus}{\Perp} \stackrel{\oplus}{\Perp}$

$\frac{\text { ๘ }}{\frac{\varepsilon}{U}}$

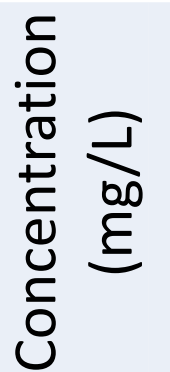




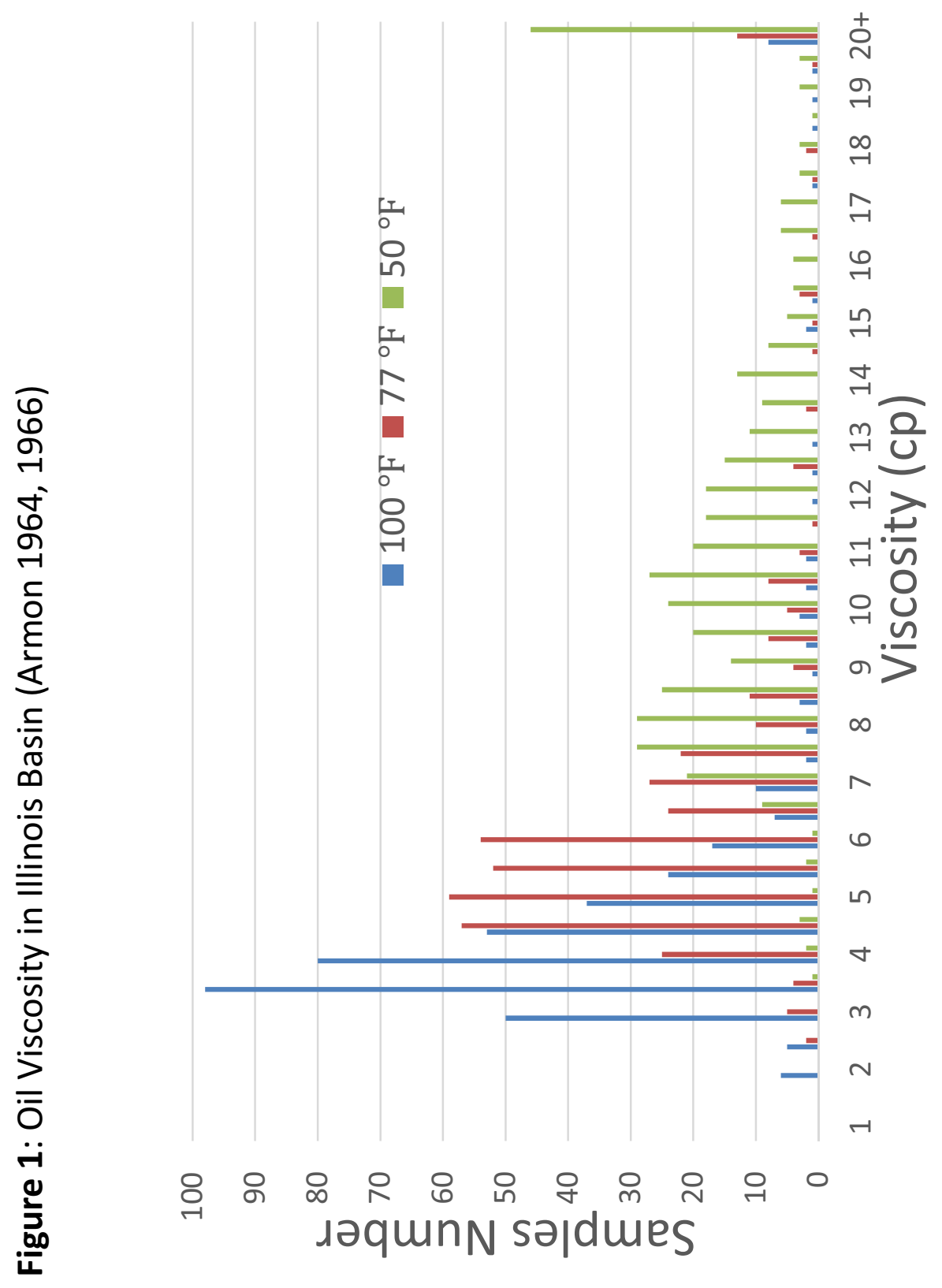



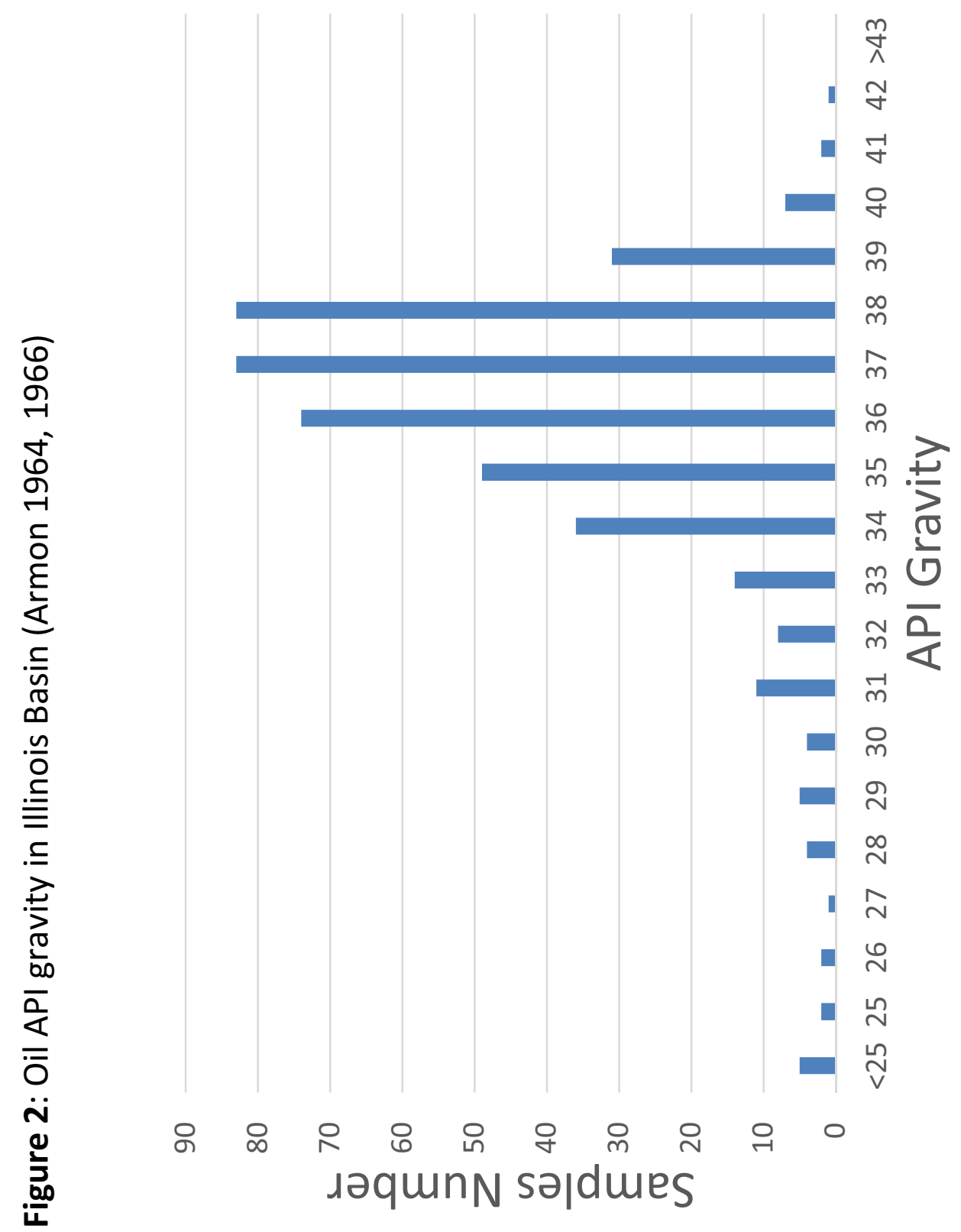

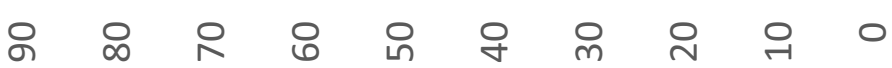
ıəqunN sojdures 


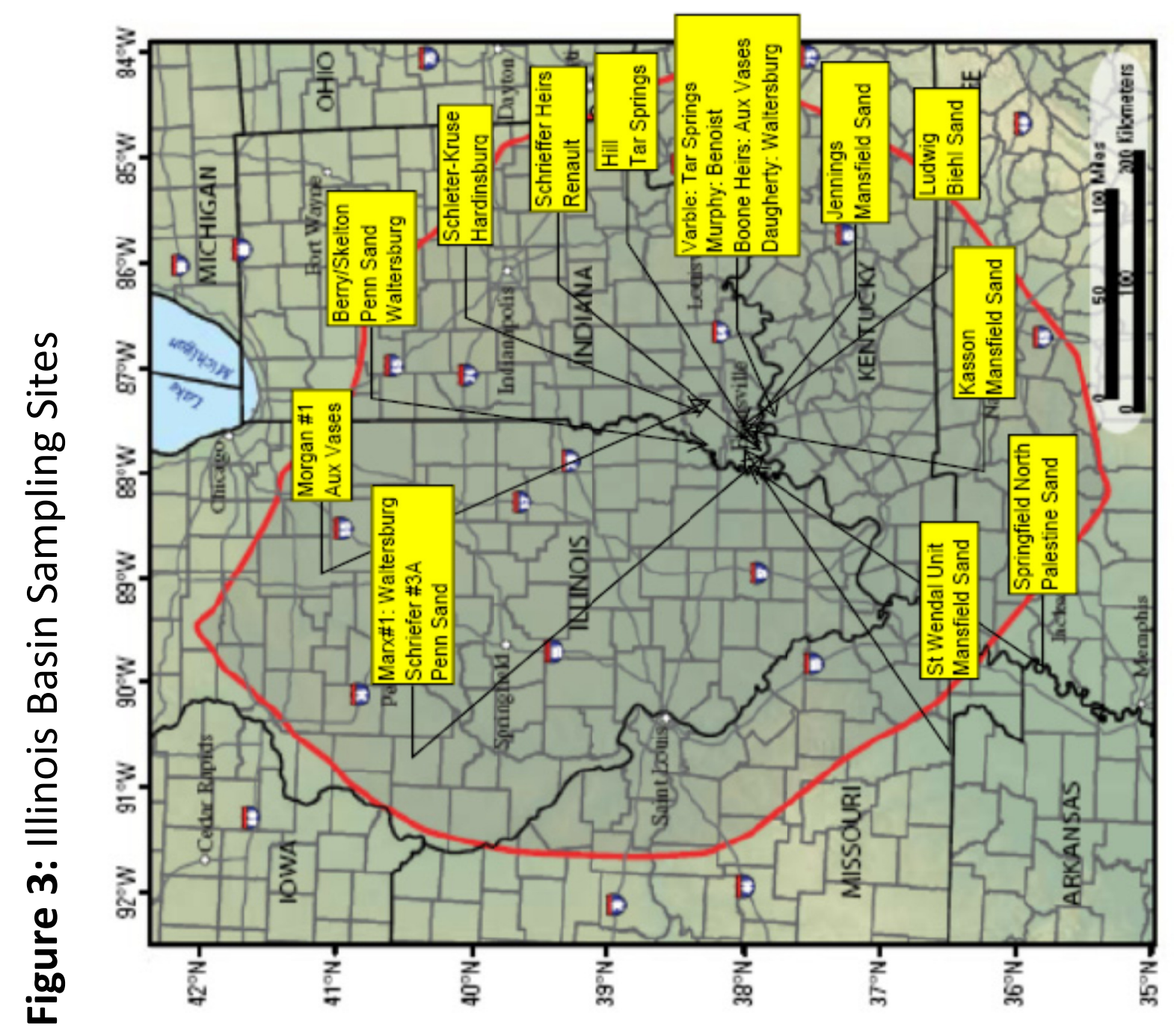




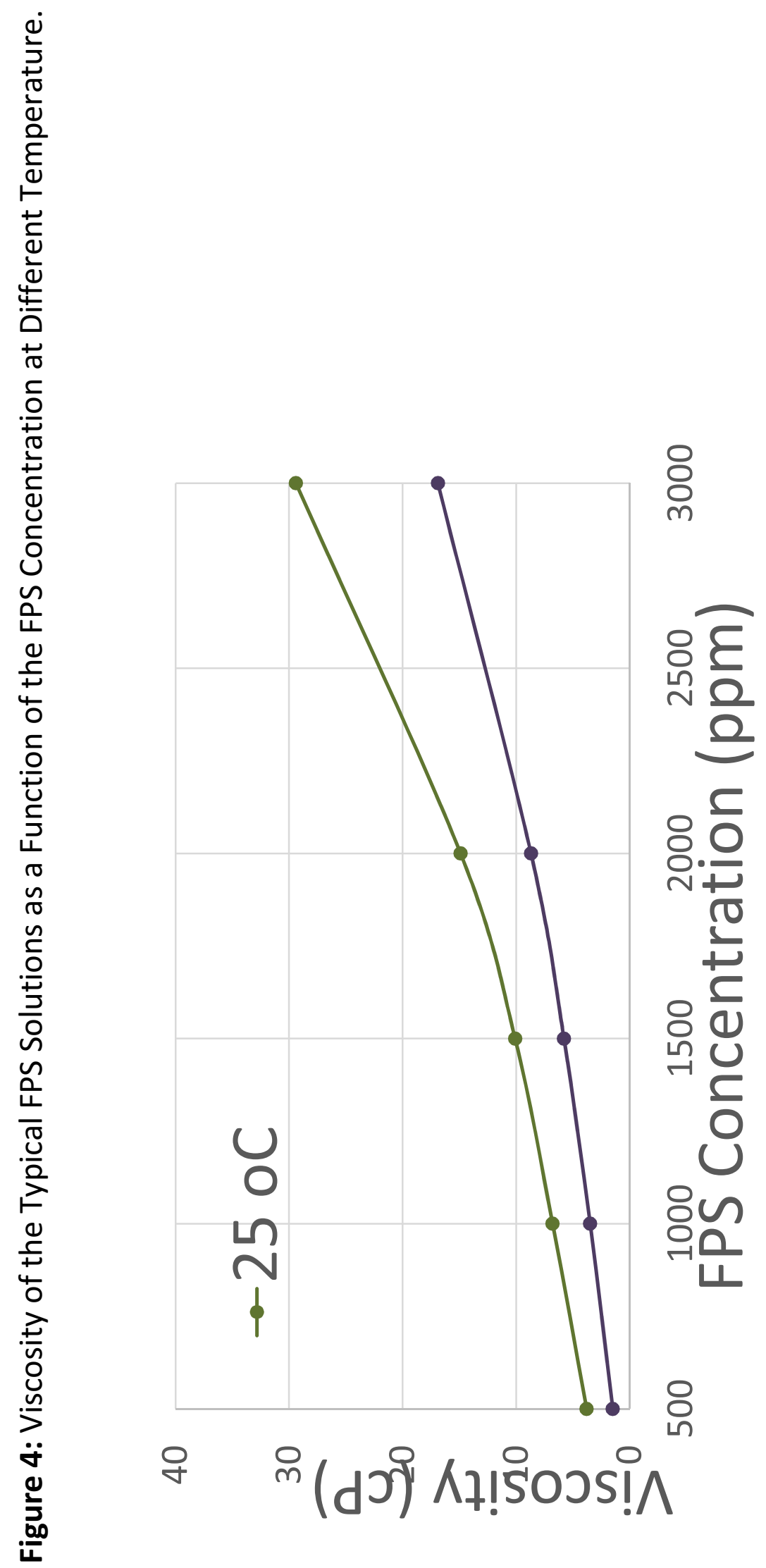



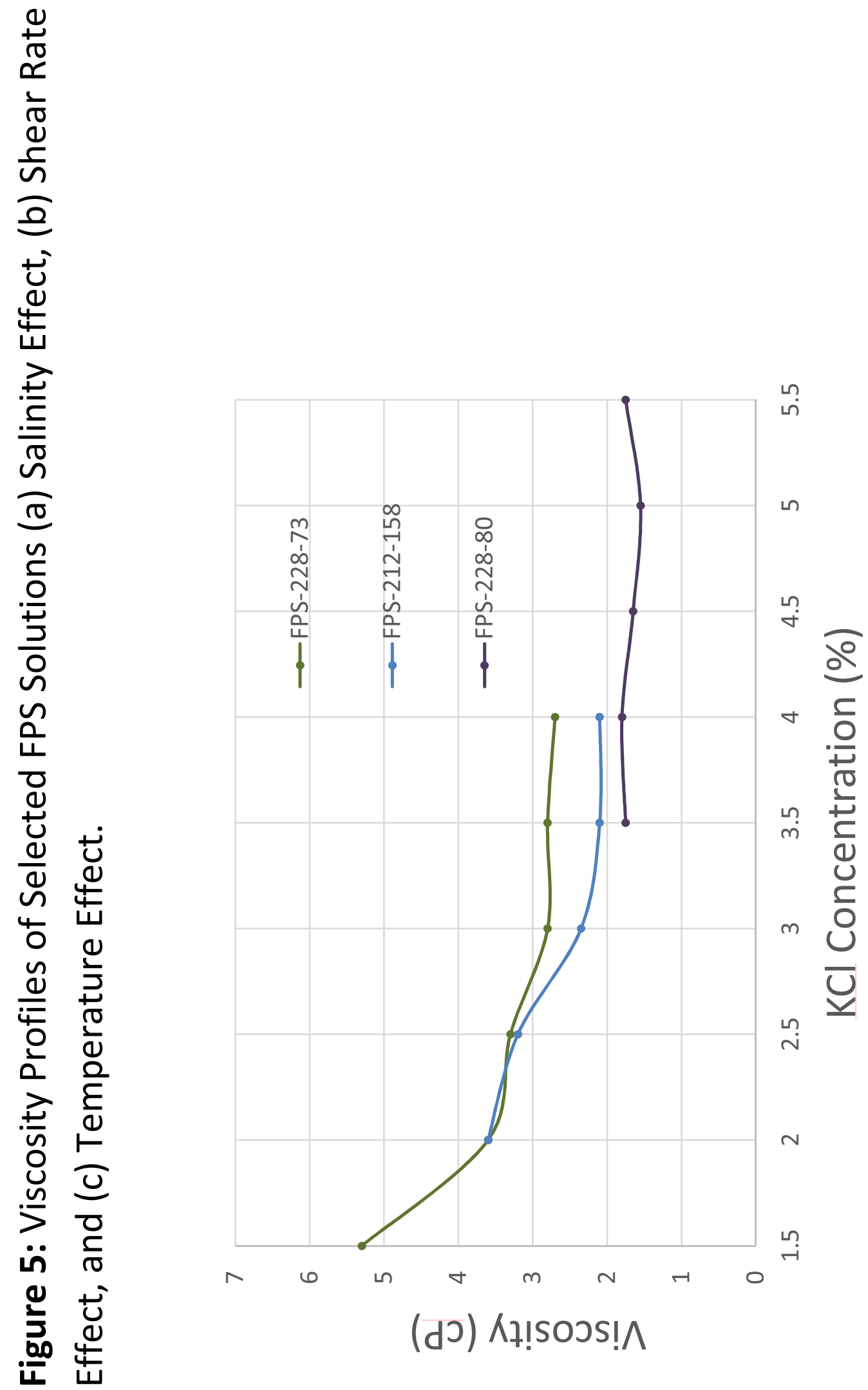


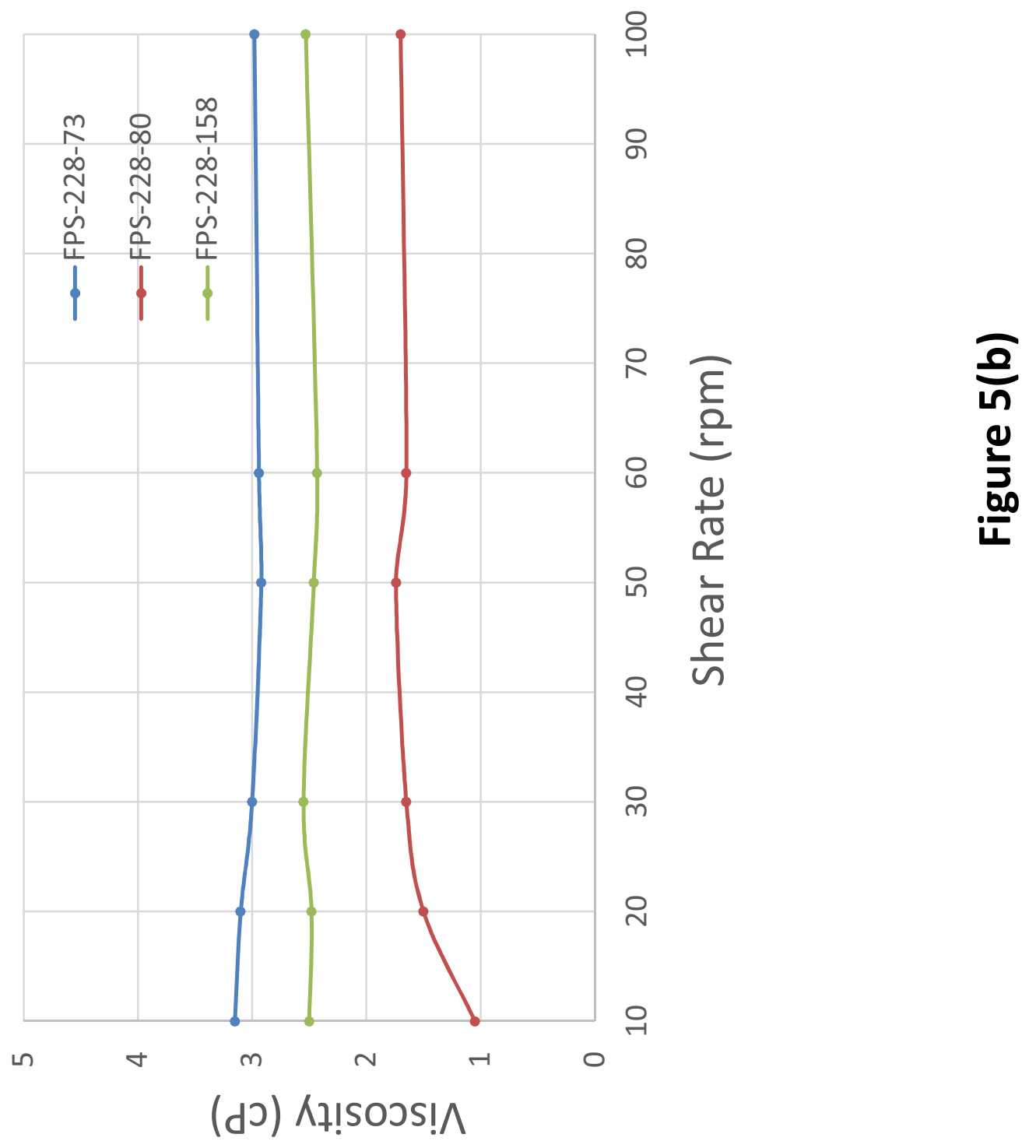




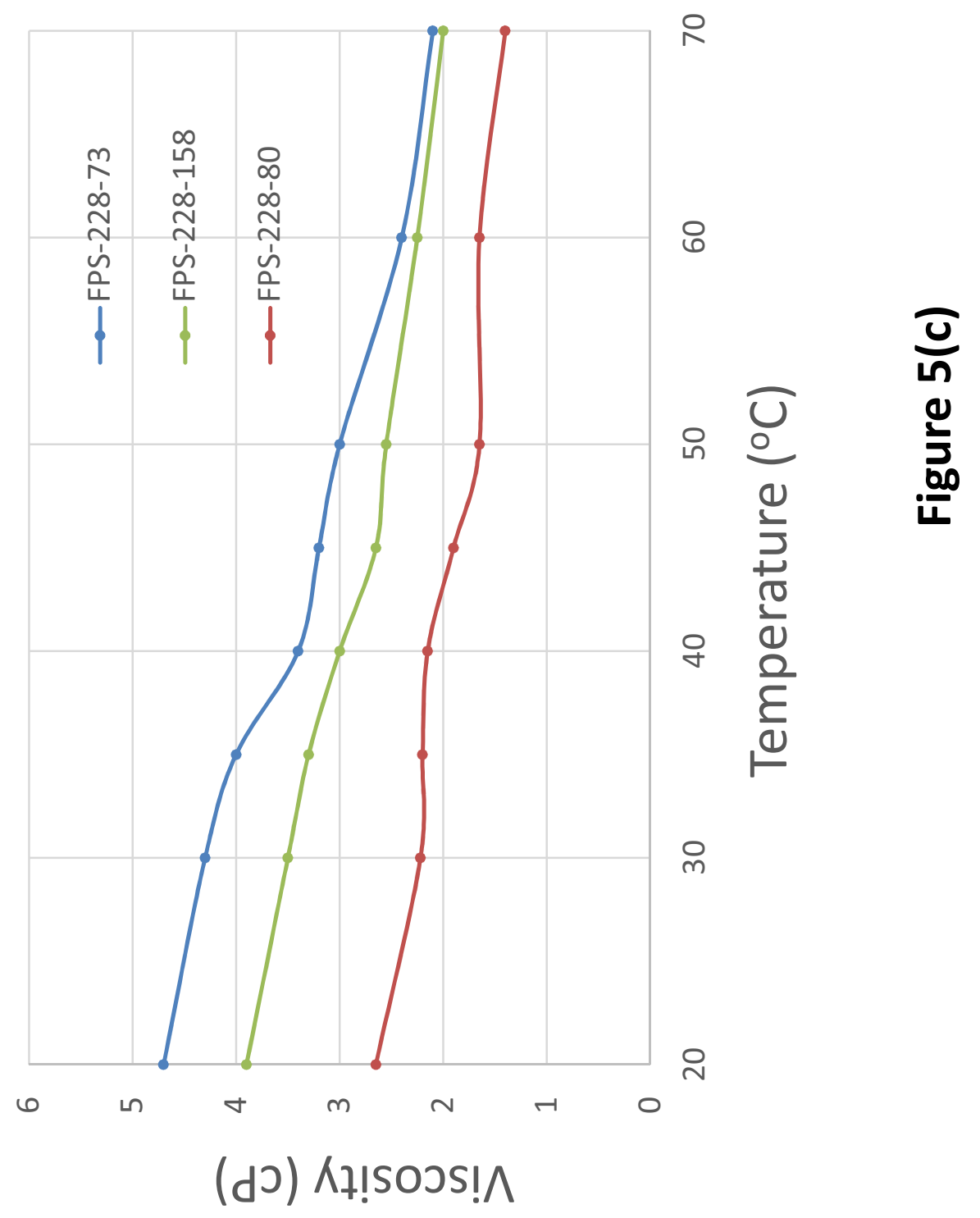



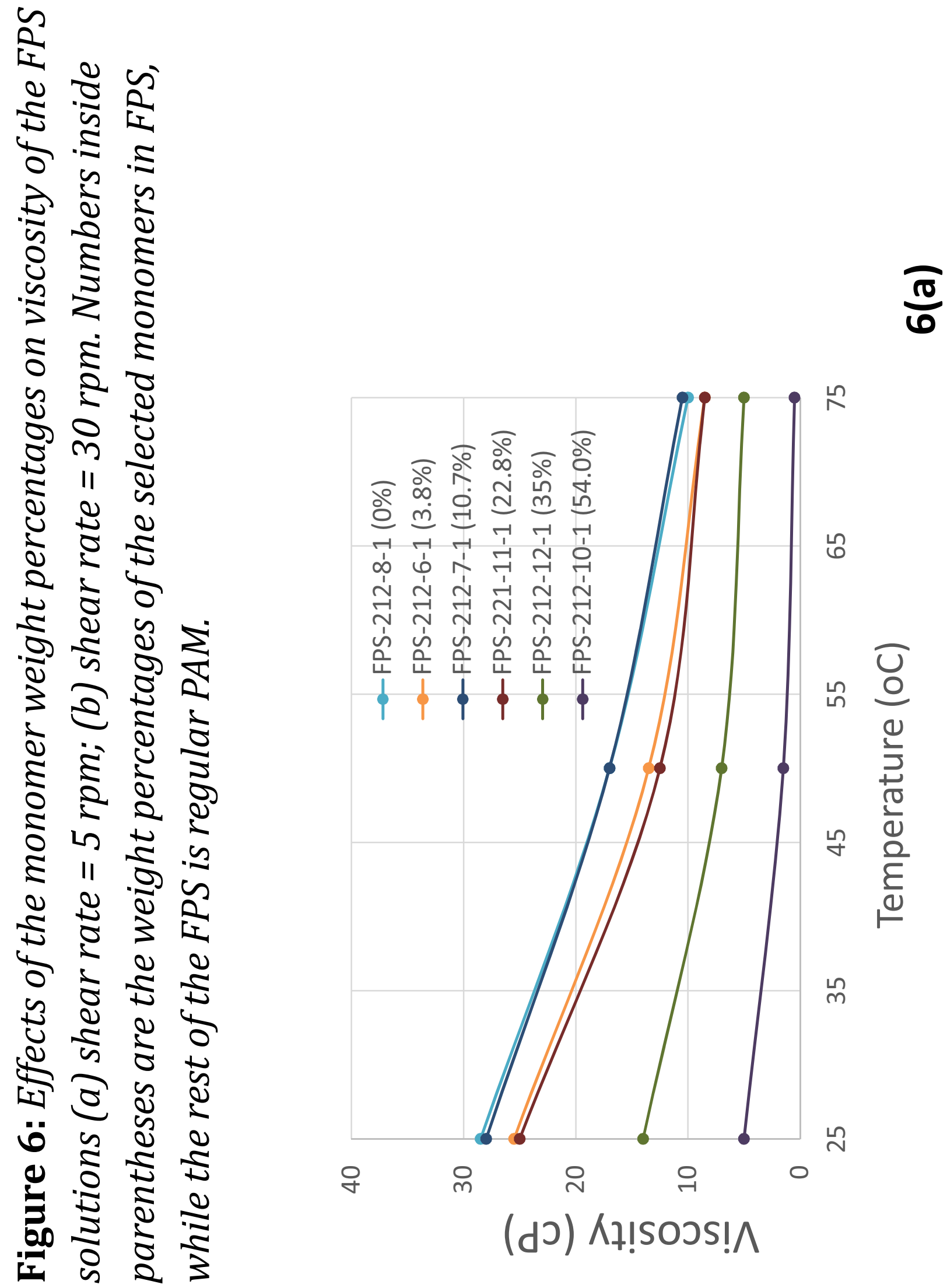


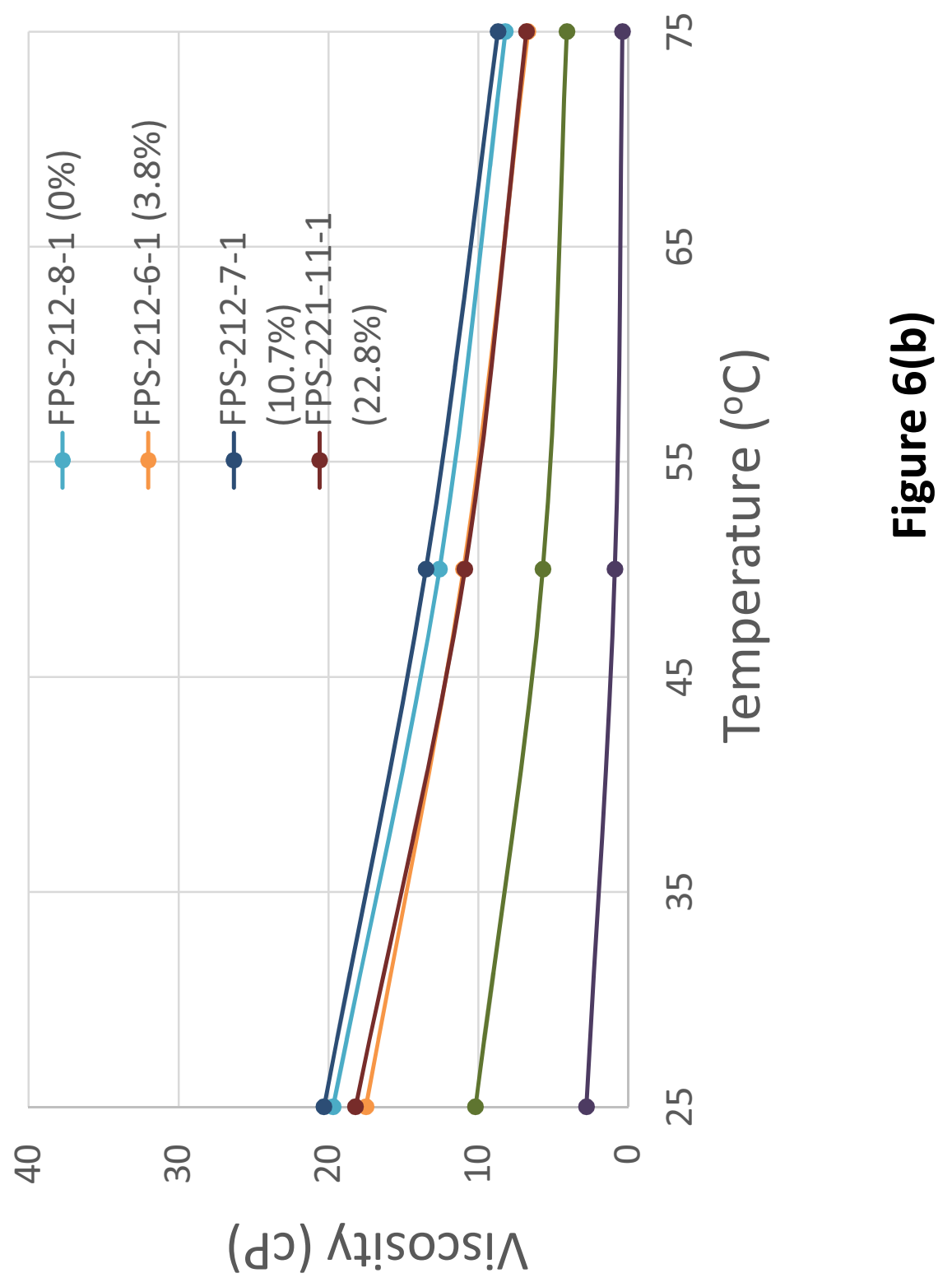



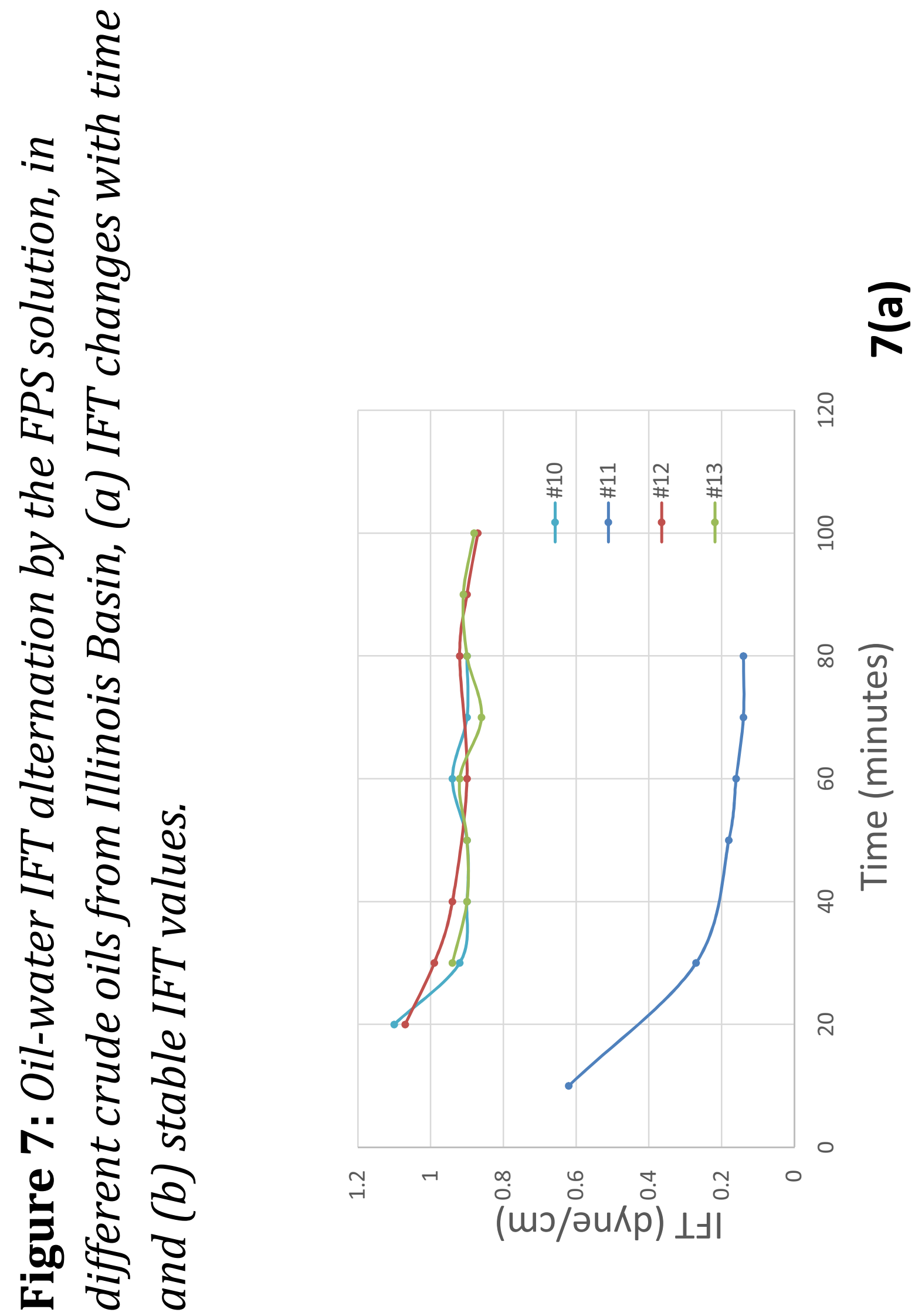


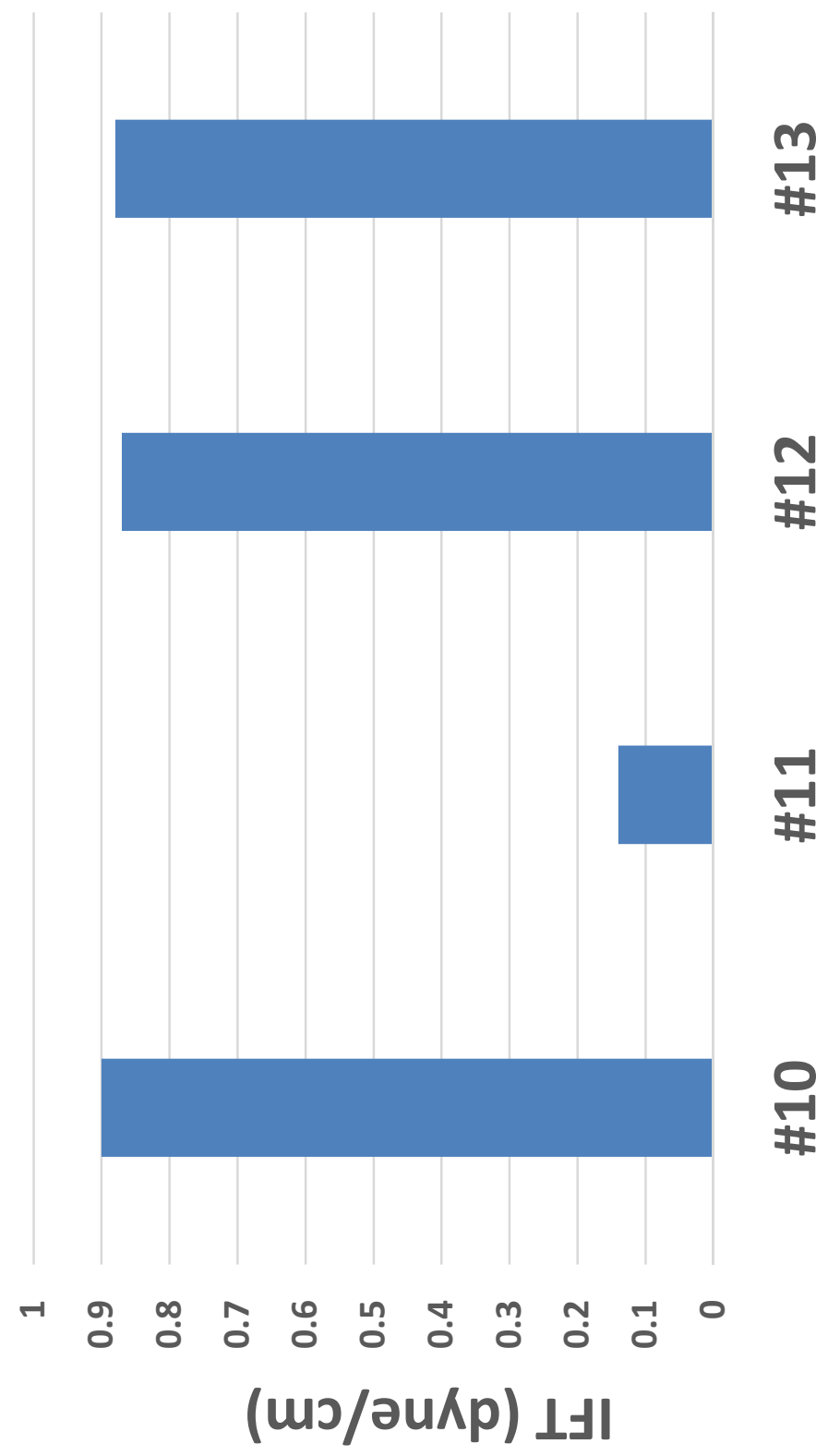

$\underbrace{\frac{0}{60}}_{\frac{0}{3}}$

\#

H

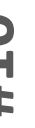




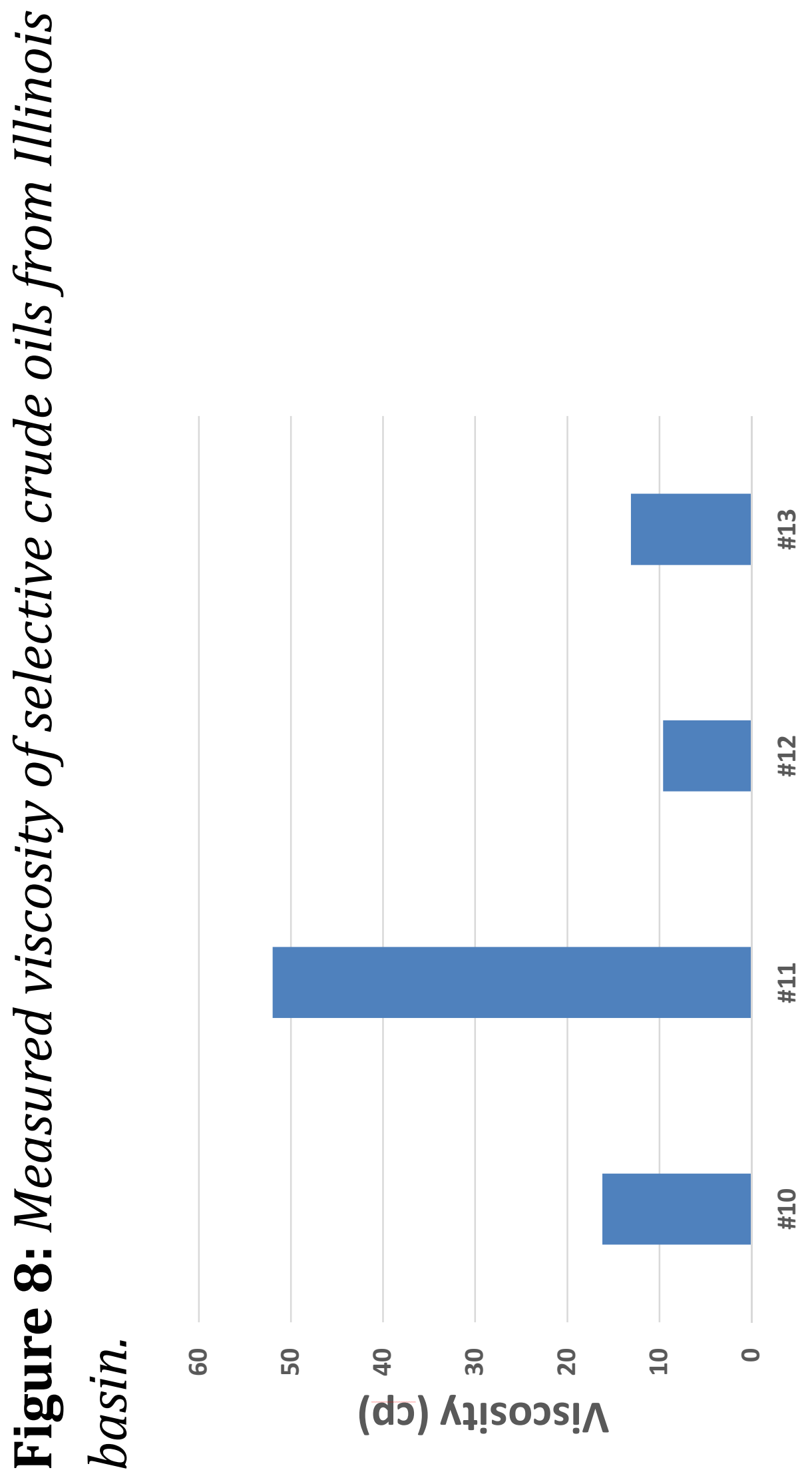




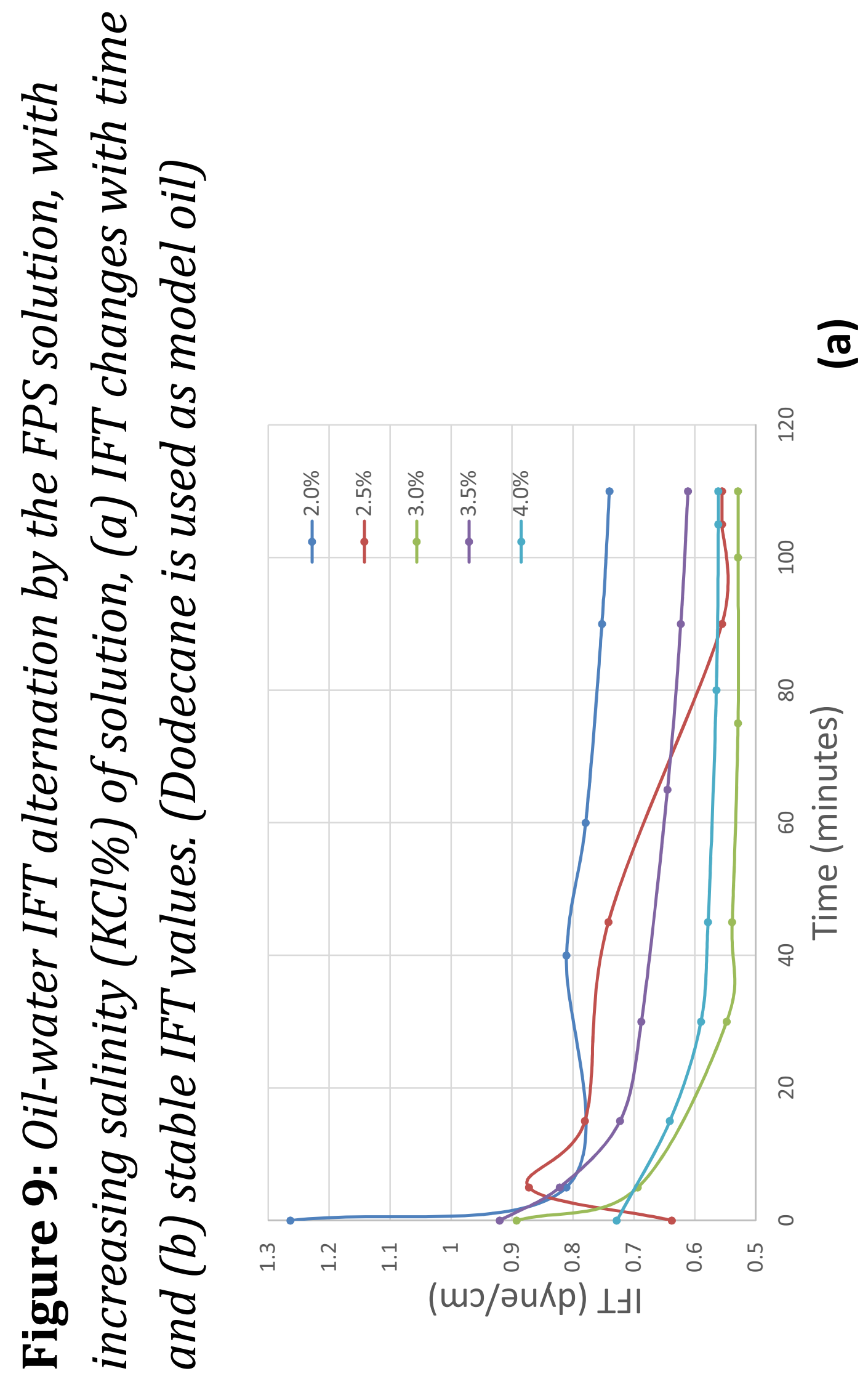




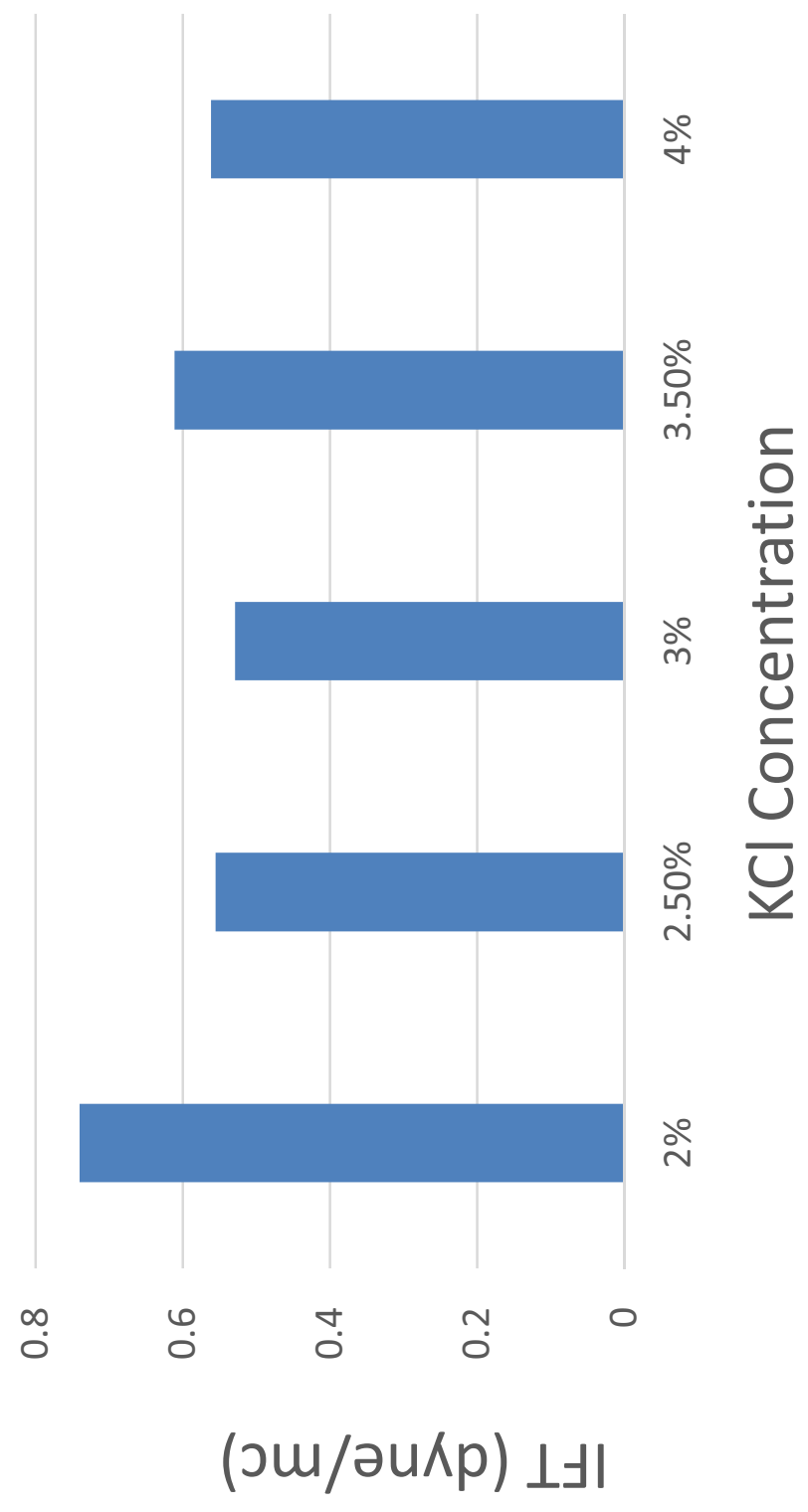

$\underline{\underline{a}}$ 

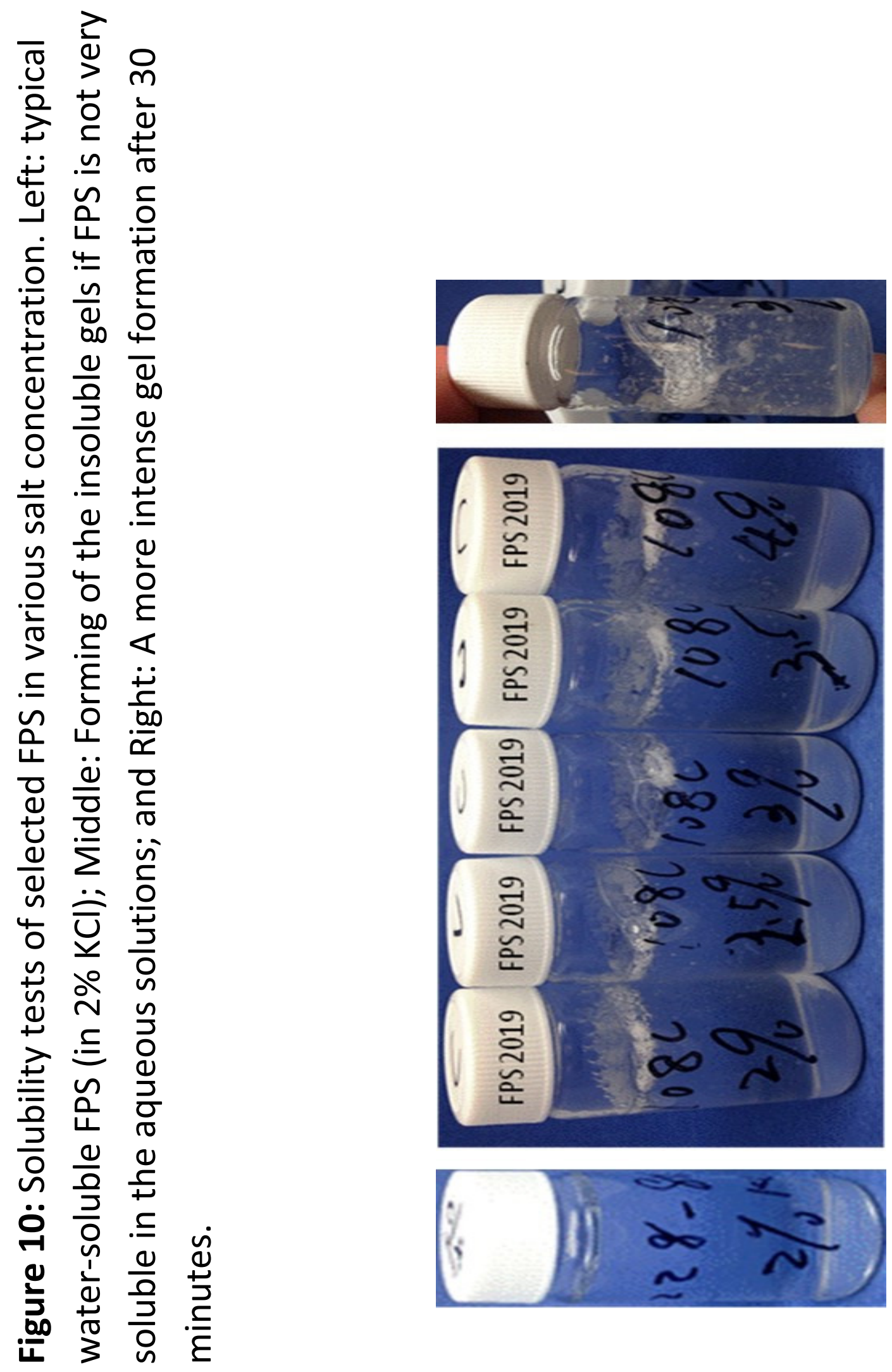

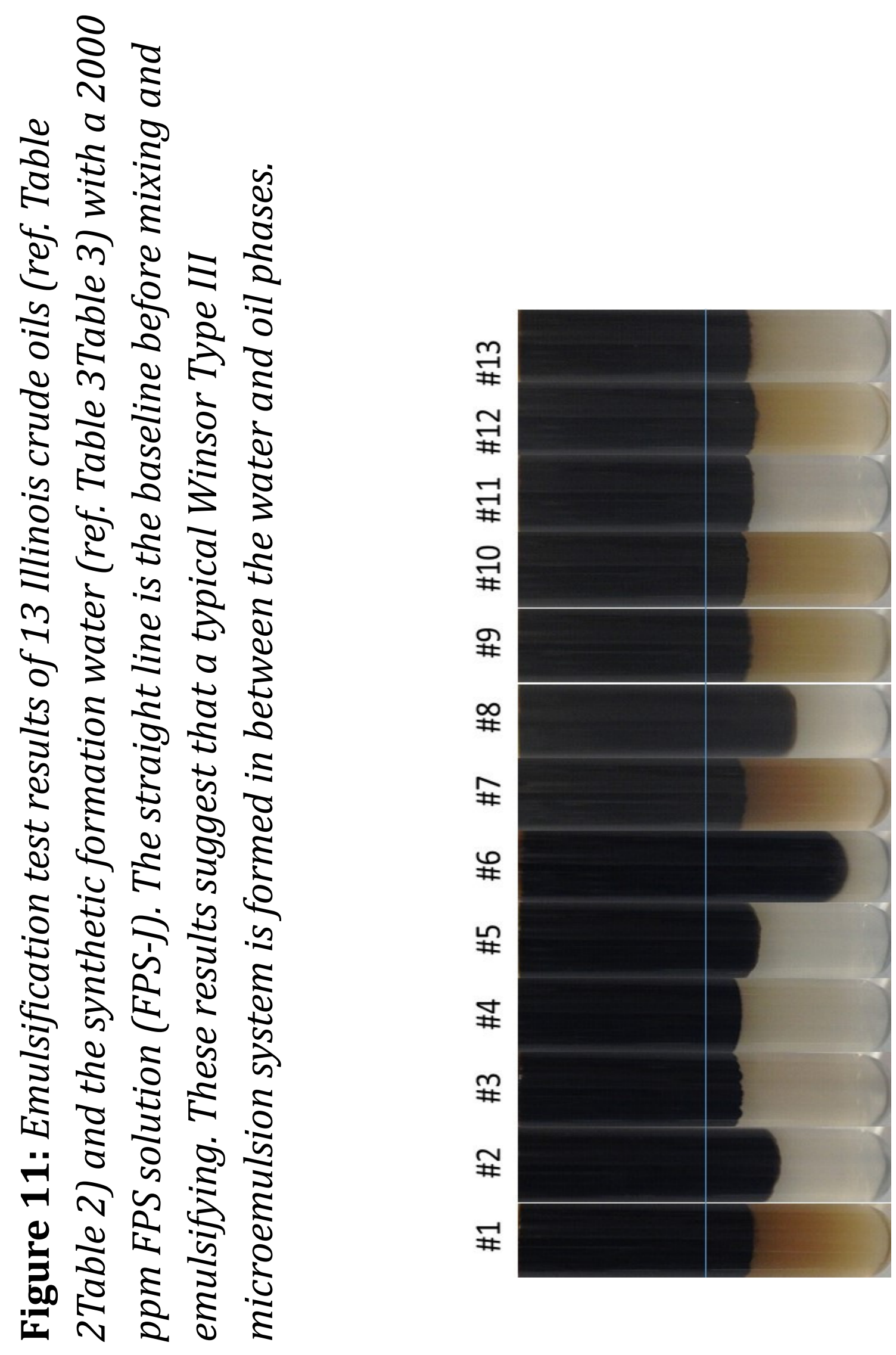


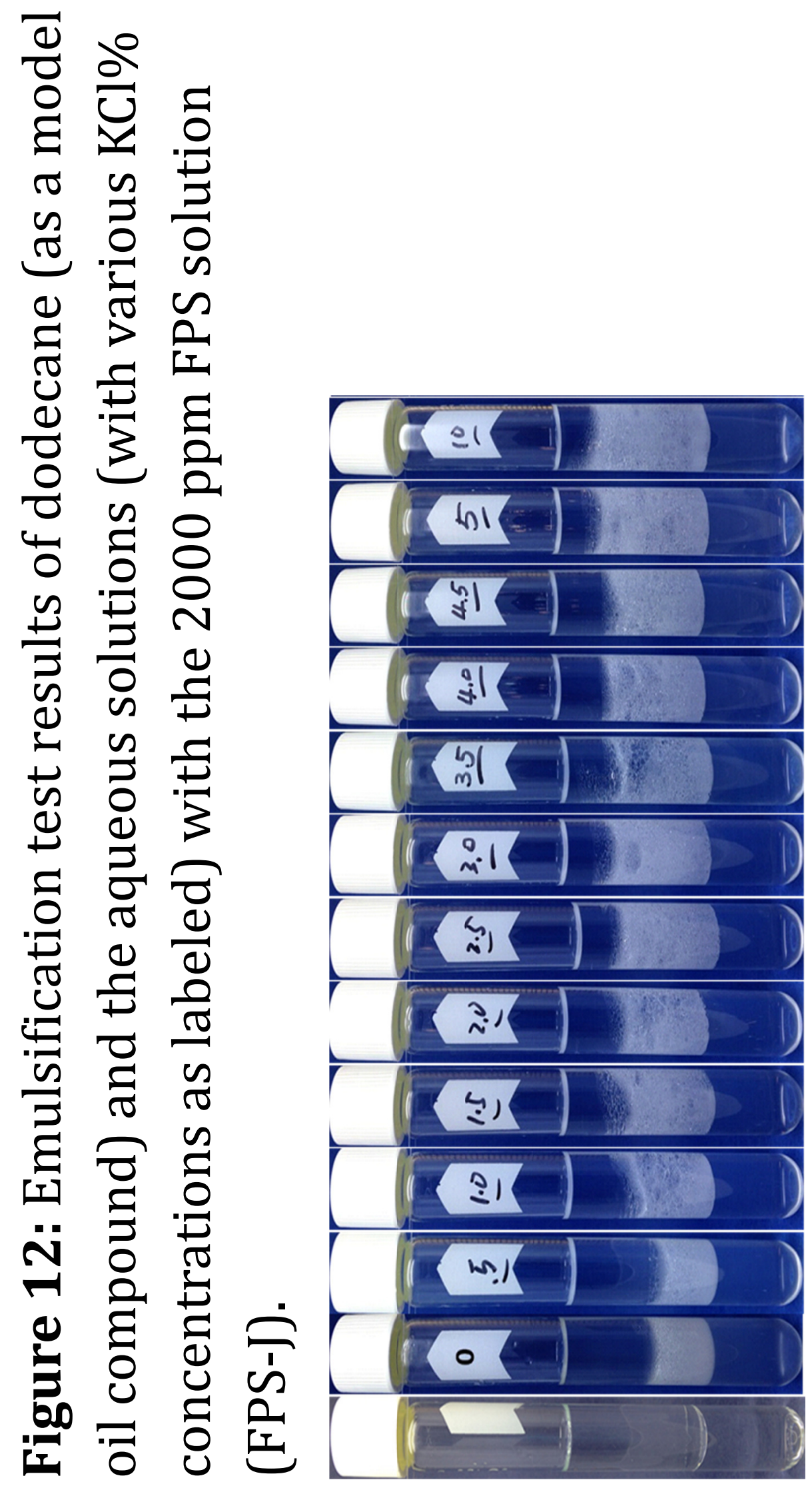



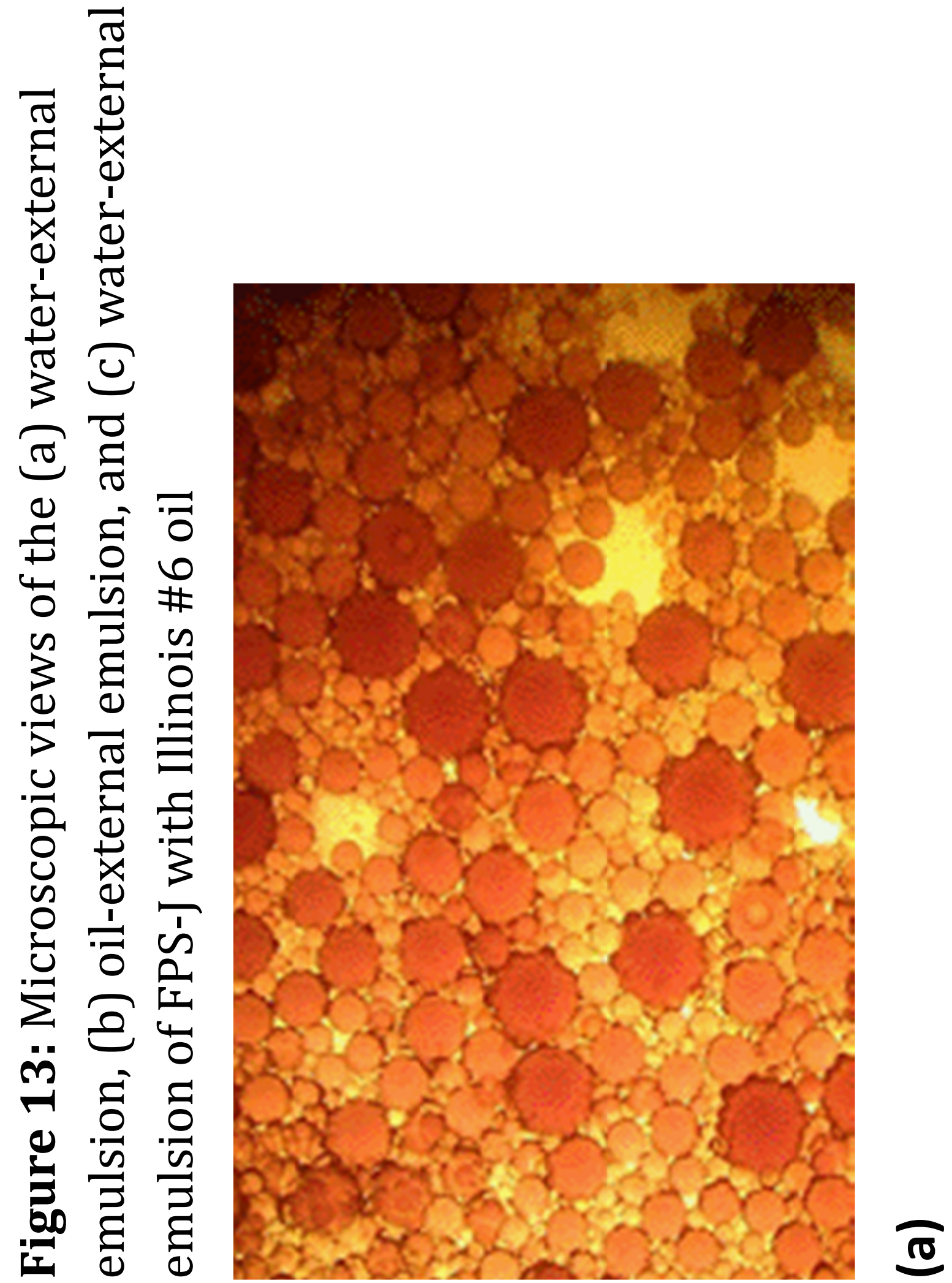


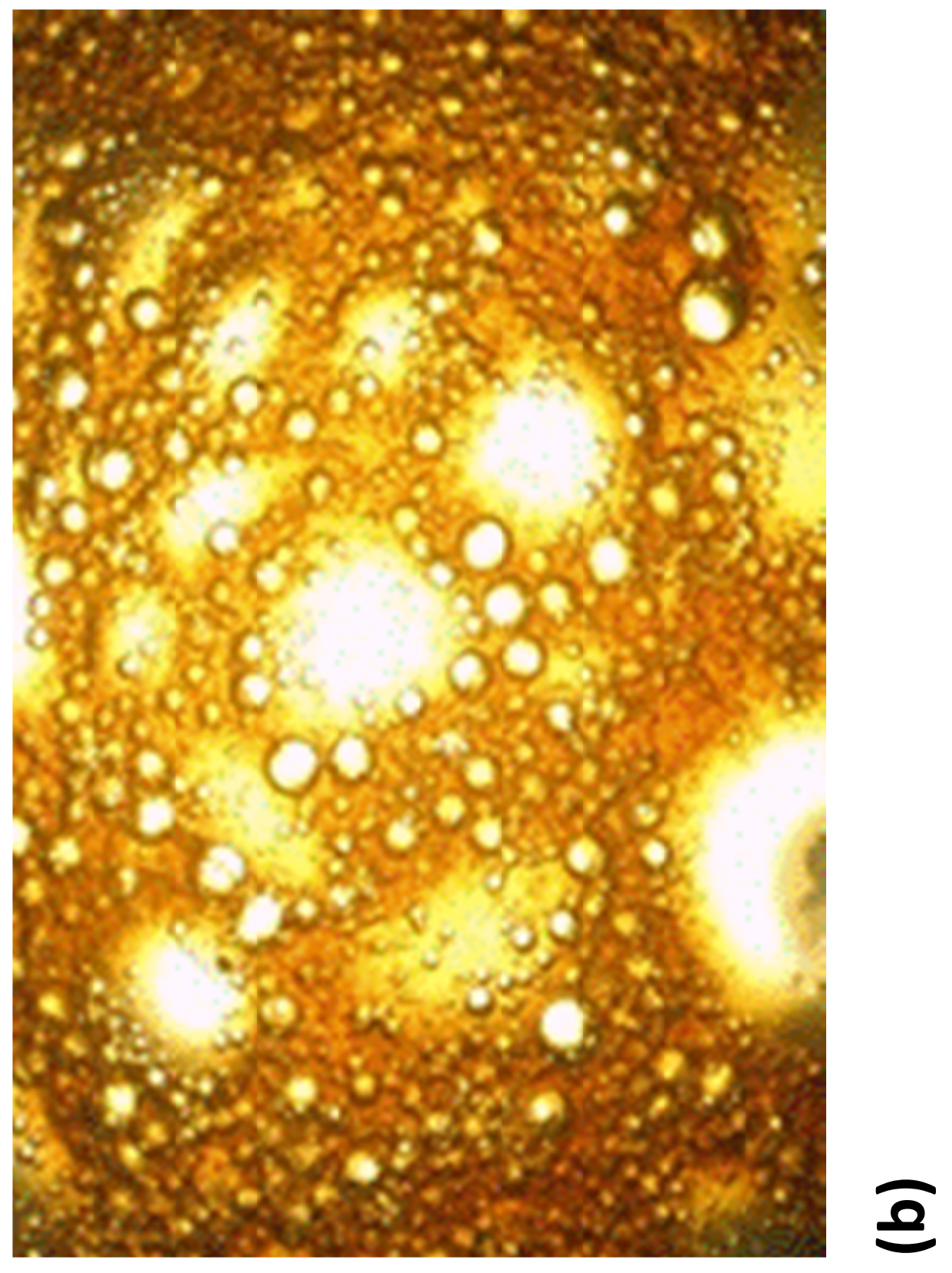




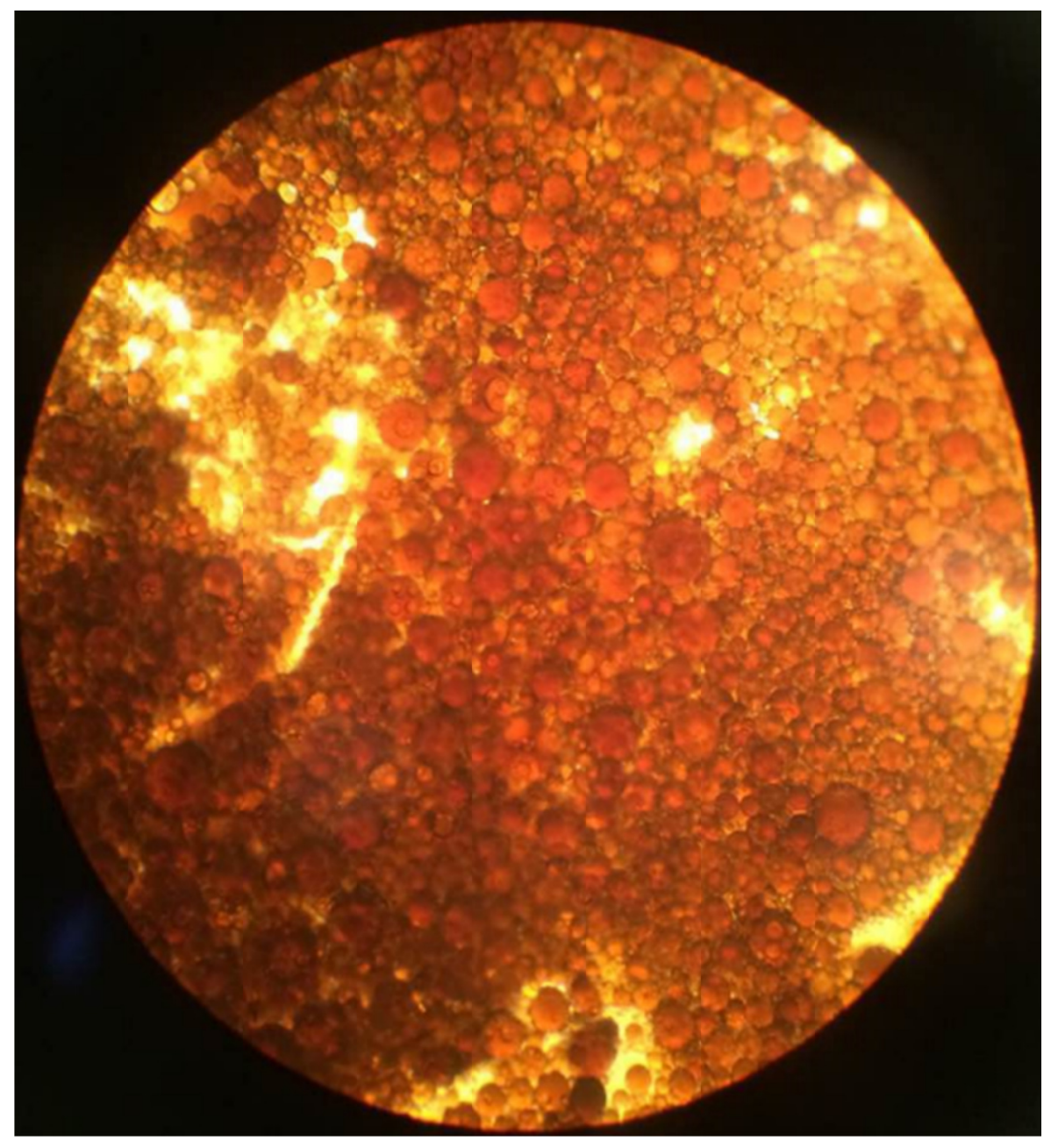

ভ 

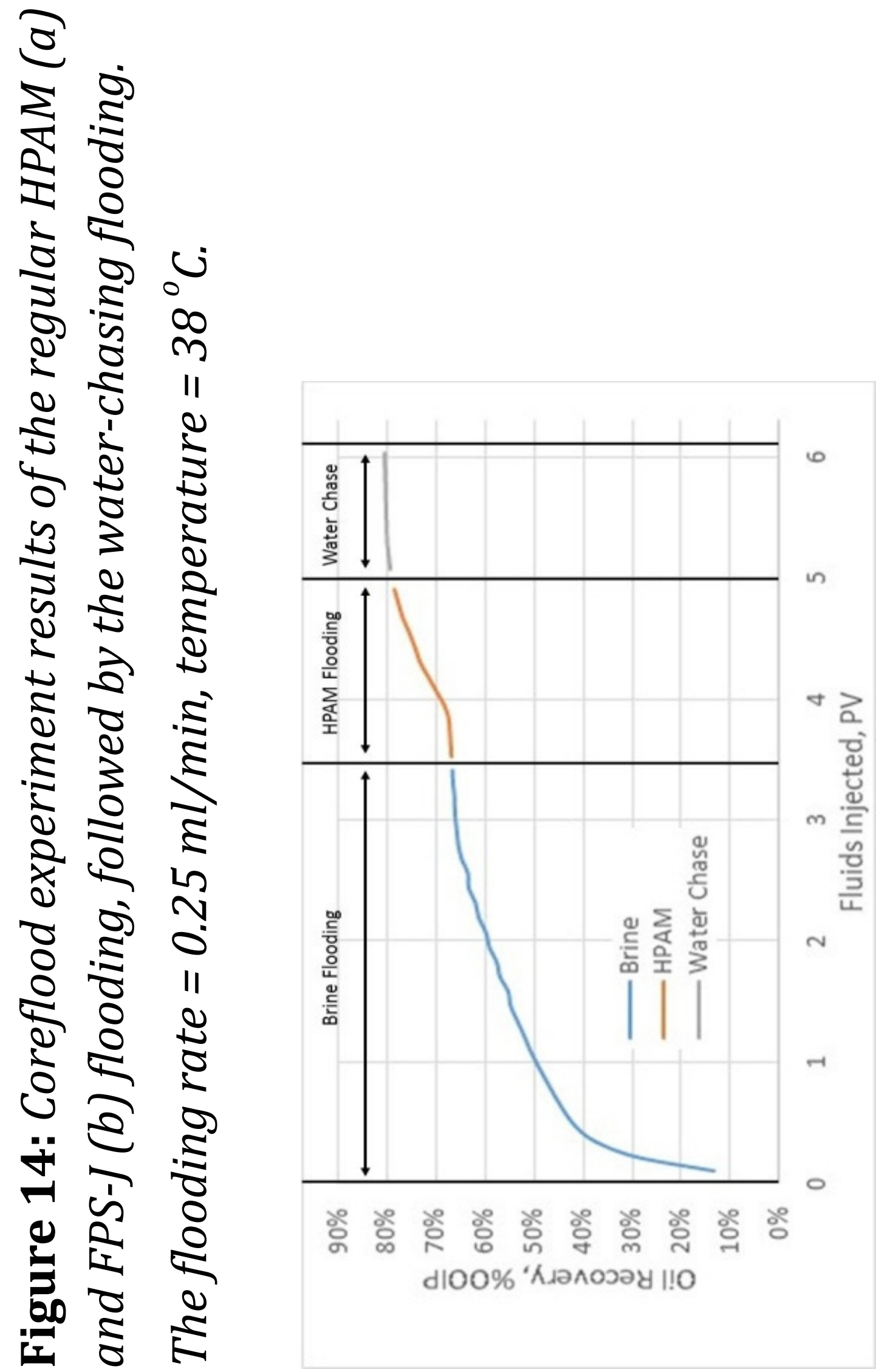


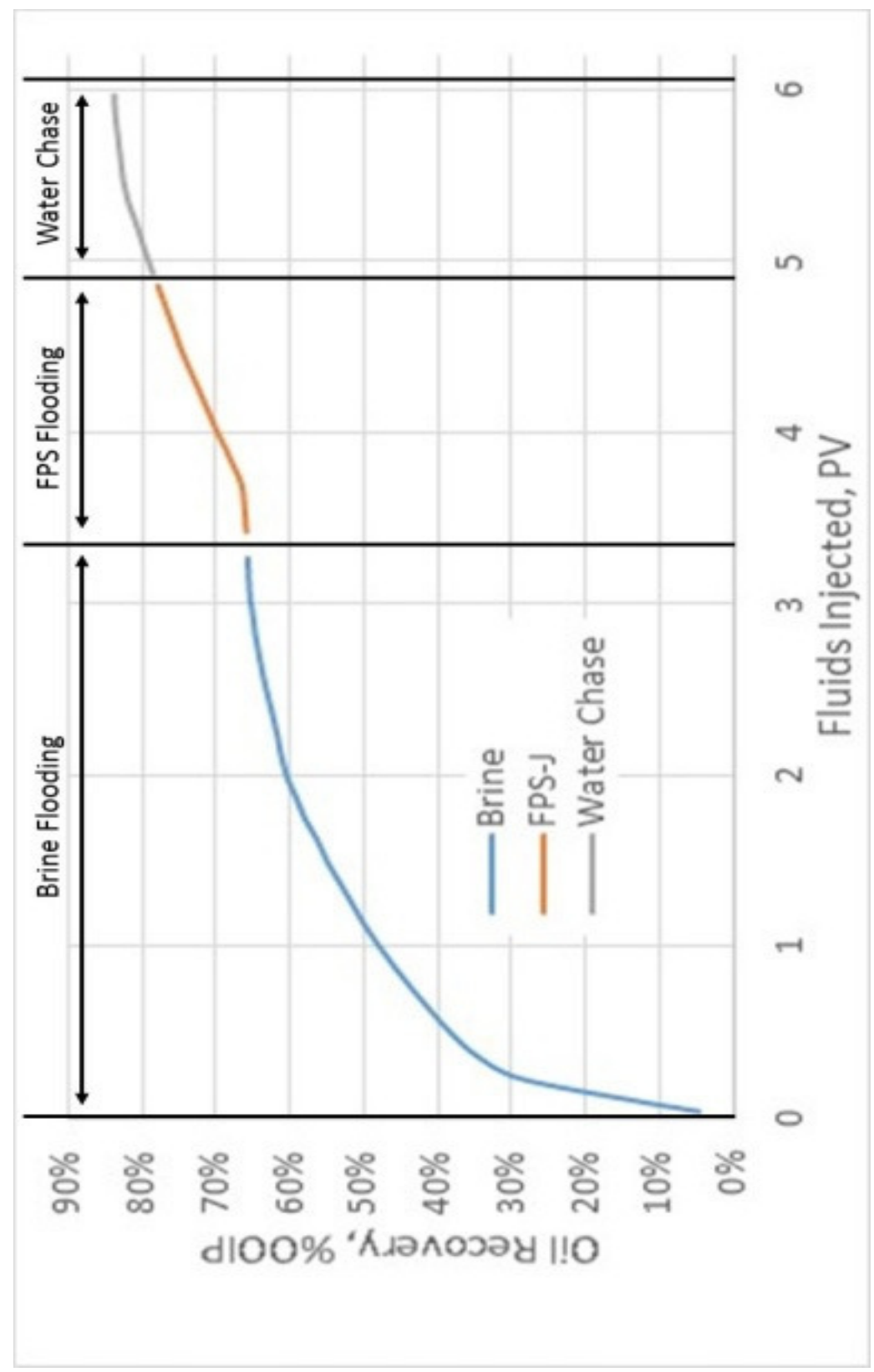

으 


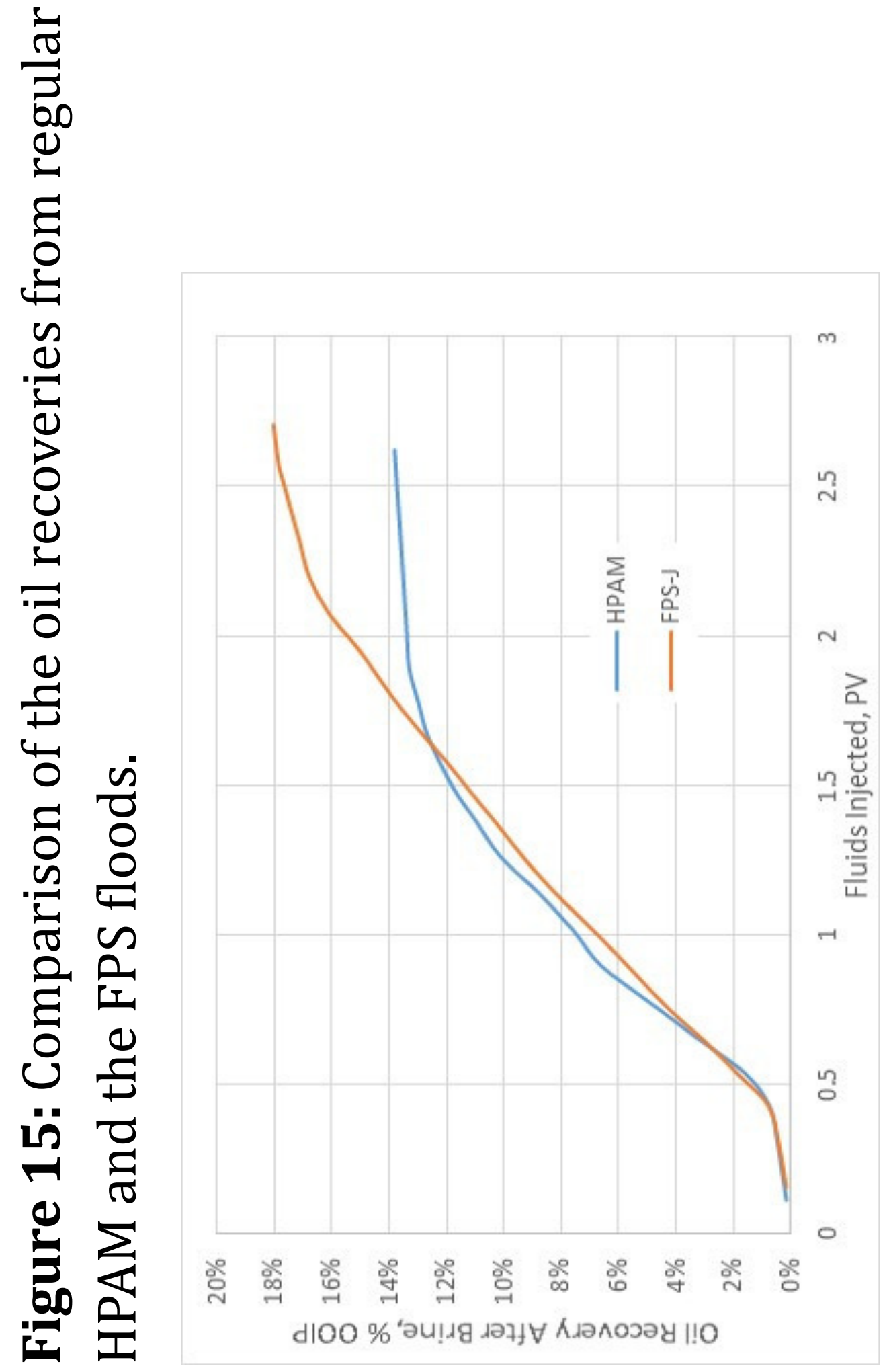




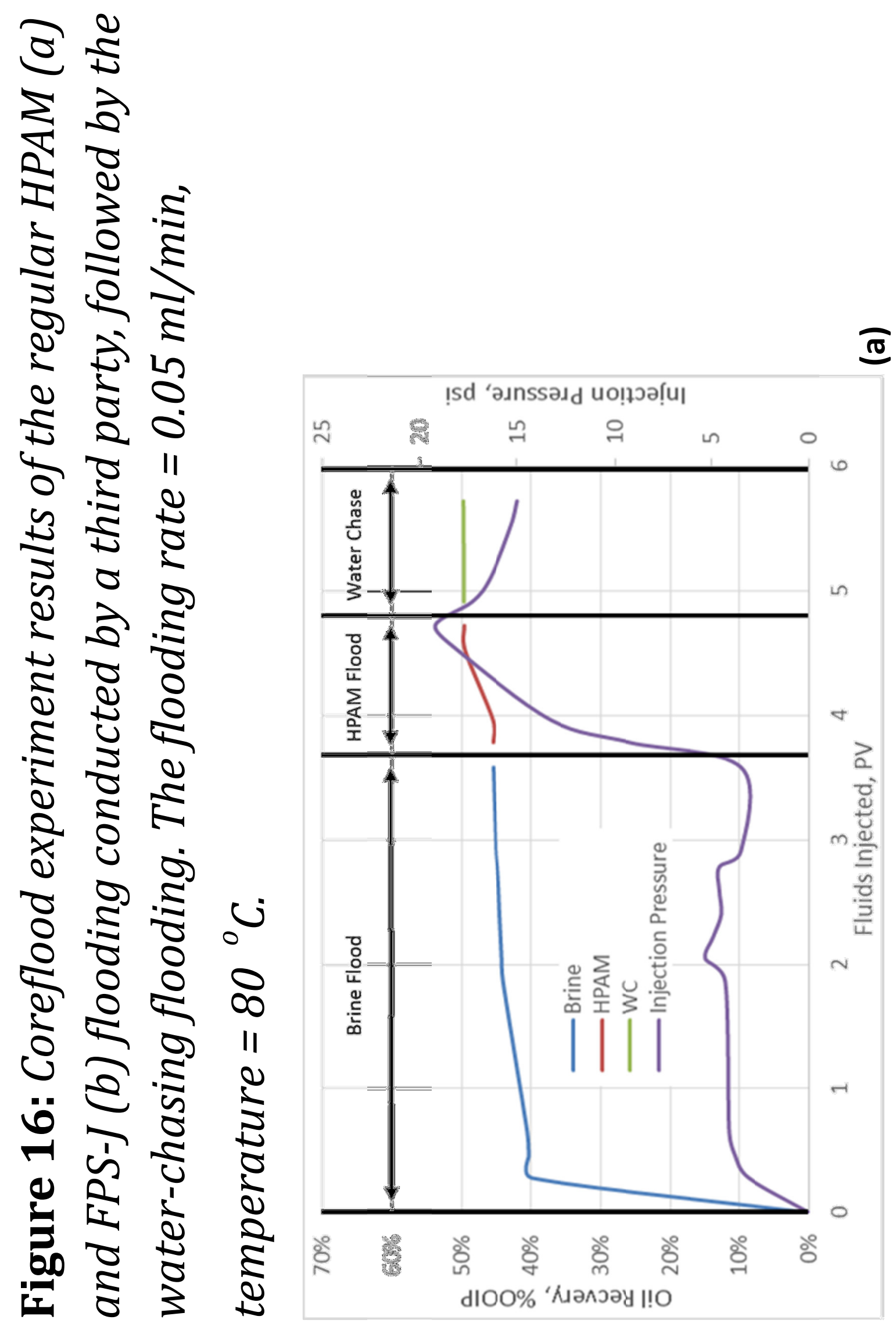




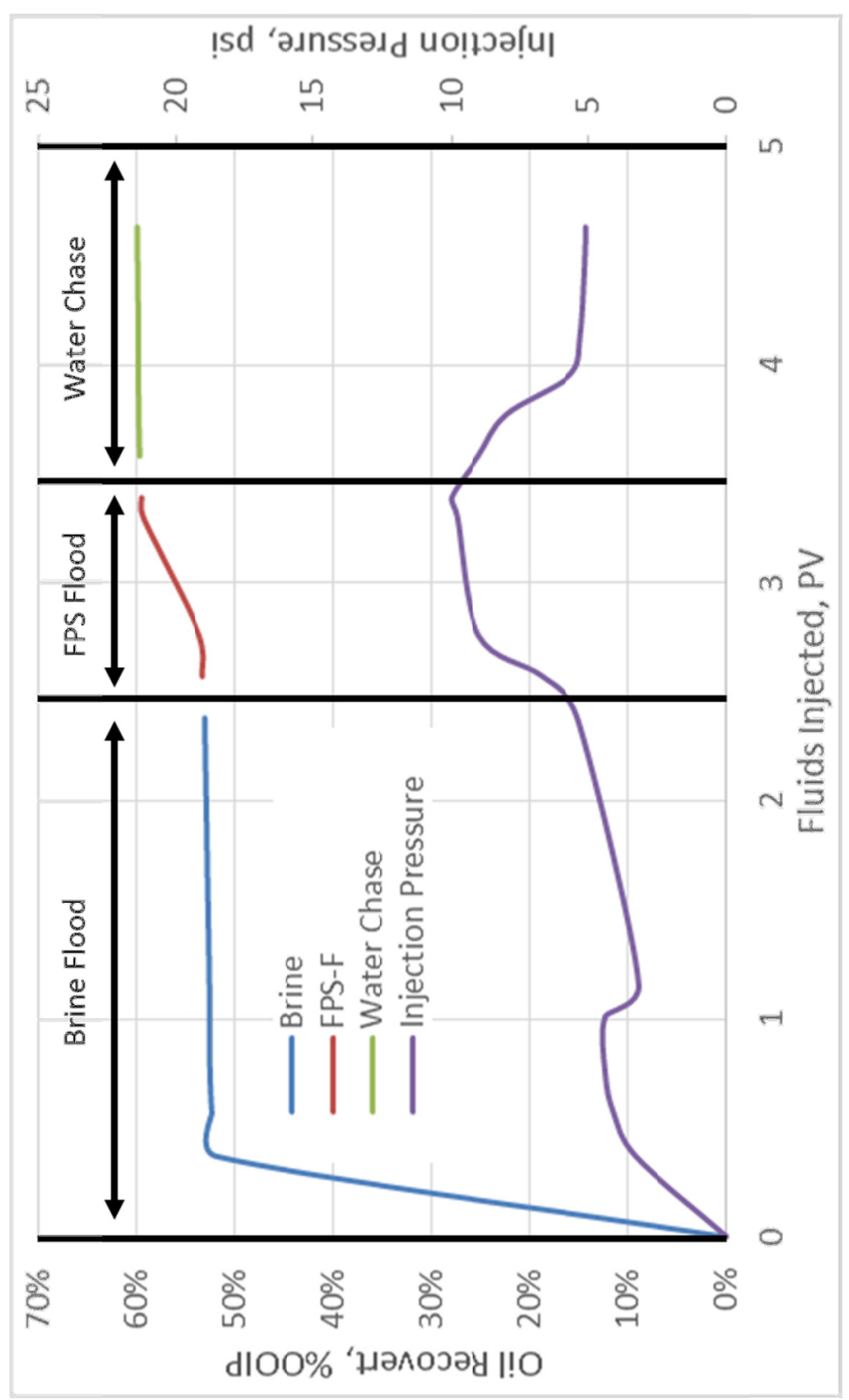

으 